OPEN ACCESS

Edited by:

José Lozano,

University of Malaga, Spain

Reviewed by:

Jaewon Ko,

Daegu Gyeongbuk Institute of Science and Technology (DGIST), South Korea

Takeshi Uemura,

Shinshu University, Japan

Doyoun Kim,

Korea Research Institute of Chemical

Technology (KRICT), South Korea

*Correspondence:

Gonzalo I. Cancino

gonzalo.cancino@umayor.cl

Specialty section:

This article was submitted to

Signaling,

a section of the journal

Frontiers in Cell and Developmental

Biology

Received: 28 January 2021 Accepted: 17 November 2021

Published: 13 December 2021

Citation:

Cornejo F, Cortés BI, Findlay GM and Cancino Gl (2021) LAR Receptor Tyrosine Phosphatase Family in Healthy and Diseased Brain. Front. Cell Dev. Biol. 9:659951. doi: 10.3389/fcell.2021.659951

\section{LAR Receptor Tyrosine Phosphatase Family in Healthy and Diseased Brain}

\author{
Francisca Cornejo ${ }^{1}$, Bastián I. Cortés ${ }^{1}$, Greg M. Findlay ${ }^{2}$ and Gonzalo I. Cancino ${ }^{1,3 *}$ \\ ${ }^{1}$ Center for Integrative Biology, Facultad de Ciencias, Universidad Mayor, Santiago, Chile, ${ }^{2}$ MRC Protein Phosphorylation and \\ Ubiquitylation Unit, School of Life Sciences, University of Dundee, Dundee, United Kingdom, ${ }^{3}$ Escuela de Biotecnología, Facultad \\ de Ciencias, Universidad Mayor, Santiago, Chile
}

Protein phosphatases are major regulators of signal transduction and they are involved in key cellular mechanisms such as proliferation, differentiation, and cell survival. Here we focus on one class of protein phosphatases, the type IIA Receptor-type Protein Tyrosine Phosphatases (RPTPs), or LAR-RPTP subfamily. In the last decade, LAR-RPTPs have been demonstrated to have great importance in neurobiology, from neurodevelopment to brain disorders. In vertebrates, the LAR-RPTP subfamily is composed of three members: PTPRF (LAR), PTPRD (PTPS) and PTPRS (PTP $)$ ), and all participate in several brain functions. In this review we describe the structure and proteolytic processing of the LARRPTP subfamily, their alternative splicing and enzymatic regulation. Also, we review the role of the LAR-RPTP subfamily in neural function such as dendrite and axon growth and guidance, synapse formation and differentiation, their participation in synaptic activity, and in brain development, discussing controversial findings and commenting on the most recent studies in the field. Finally, we discuss the clinical outcomes of LAR-RPTP mutations, which are associated with several brain disorders.

Keywords: brain disorders, protein phosphatase, receptor protein tyrosine phosphatase (RPTP), PTPdelta, PTPsigma

\section{INTRODUCTION}

Post-translational regulation involves covalent modifications that control protein activity, with phosphorylation being the most common modification (Tonks, 2006). Since reversible phosphorylation is a major feature in cellular signaling (Cohen, 2002), dephosphorylation reactions are equally important for controlling cellular processes. The complementary roles of protein kinases and protein phosphatases have been underlined by studies showing that protein kinases mediate the amplitude of a signal, whilst protein phosphatases may control its rate and duration (Tonks, 2006). Eukaryotic protein phosphorylation typically occurs on serine, threonine or tyrosine residues and protein phosphatases are often classified according to the residue that they dephosphorylate and/or the homology of their catalytic domain (Liberti et al., 2013; Chen et al., 2017). Protein tyrosine phosphatases are classified in two families; the receptor protein tyrosine phosphatase (RPTP) and the non-receptor tyrosine phosphatases family, which play important roles in intercellular communication and intracellular signal transduction (Cohen, 2002; Chen et al., 2017).

RPTPs were discovered in 1988 (Tonks et al., 1988), and have been increasingly studied because they not only participate in cellular signaling through their phosphatase activity, but also by acting as adhesion molecules often independently of their catalytic domains (Young et al., 2021). Amongst these dual-function molecules, type IIA RPTPs arise as important modulators of several cellular 


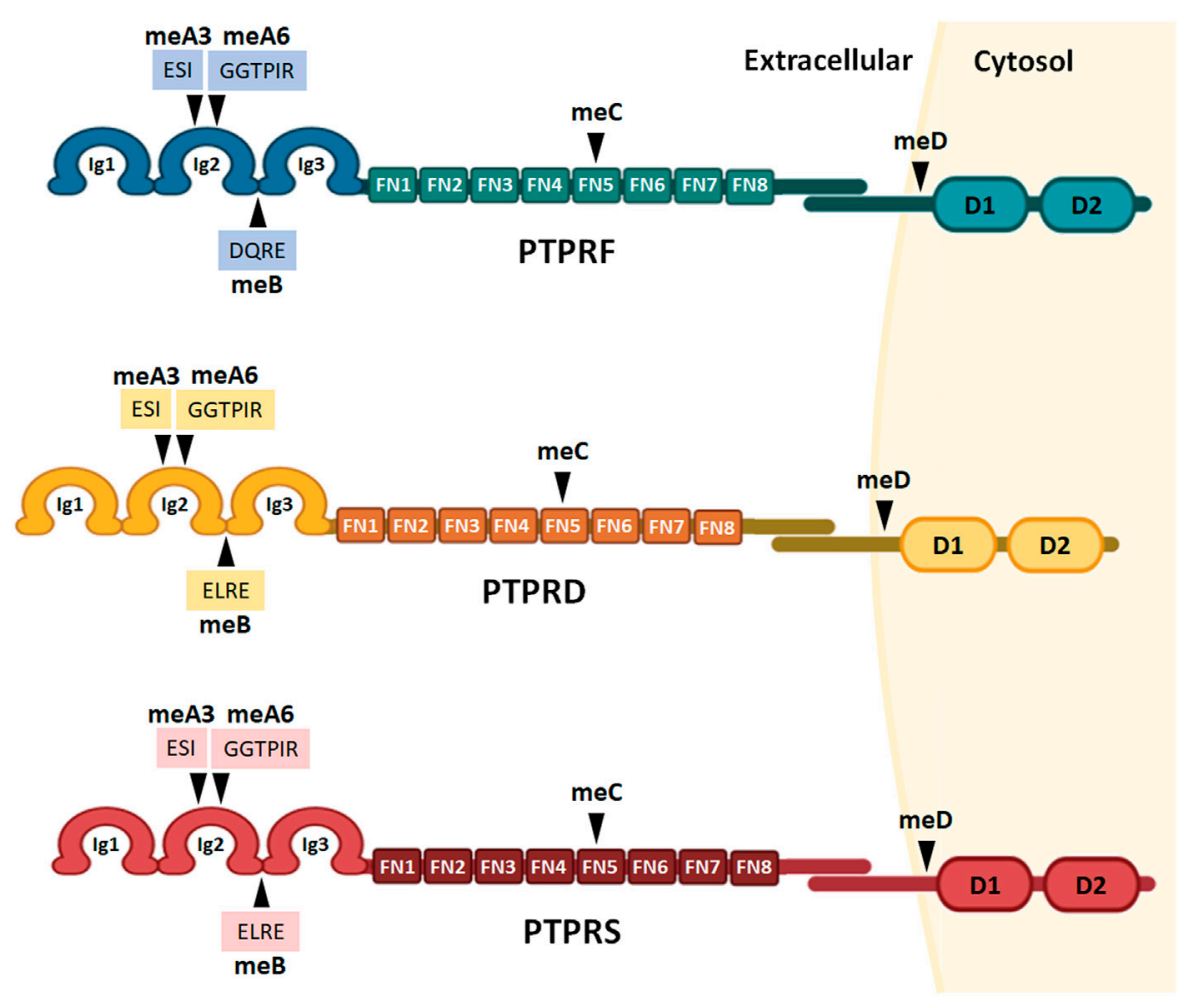

FIGURE 1 | LAR-RPTPs protein structure and alternative splicing sites. The structure of all three receptors consists of an extracellular portion of three Ig-like domains and eight extracellular fibronectin type III domains, a transmembrane region, and two intracellular protein tyrosine phosphatase domains: a membrane proximal catalytically active domain and a membrane distal domain with no phosphatase activity. Alternative splicing sites are marked as mini-exons meA-D, and the aminoacidic sequences of meA3, meA6 and meB are shown. Ig: Ig-like domains; FN: fibronectin type III domains; D1 and D2: phosphatase domains.

processes within the brain, acting as signaling and adhesion molecules. Type IIA RPTPs, also known as Leukocyte common Antigen-Related RPTP (LAR-RPTP) subfamily, are integral membrane proteins which regulate the activation of several signaling pathways by modulating tyrosine phosphorylation (Tonks, 2006; Coles et al., 2015). Indeed, a phospho-proteomic study in mouse embryonic cells revealed that activity of one member of the LAR-RPTP subfamily, PTPRF (LAR), regulates the phosphorylation state of at least 205 different proteins (Sarhan et al., 2016b). This illustrates the importance of LAR-RPTPs catalytic activity in regulating essential and diverse cellular processes such as protein synthesis and degradation, cytoskeleton organization, cell adhesion and migration, and protein transport among others (Sarhan et al., 2016b).

In the recent years, several studies have shown that LARRPTPs have important roles in the regulation of biological processes within the brain, from neural development to synaptic function and differentiation (reviewed in Chagnon et al., 2004; Tonks, 2006; Takahashi and Craig, 2013; Um and Ko, 2013; Stoker, 2015; Han et al., 2016; Won and Kim, 2018), which illustrates the importance of LAR-RPTPs in the regulation of several neural signaling pathways, and the detrimental effects that could induce its impaired expression over a wide number of essential brain processes. Therefore, we will focus on LAR-RPTPs function in the mammalian brain, reviewing the main evidence for their participation in neurobiological processes. We will discuss recent studies that suggest a secondary role for LARRPTPs in synapse development in mammals, in contrast to previous studies that assigned them a fundamental role in synaptogenesis. Also, we will summarize LAR-RPTPs participation in the etiology of neurological and psychiatric disorders, highlighting the importance of studying LAR-RPTPs as potential therapeutical targets for brain diseases.

\section{LAR-RPTP TYPES AND STRUCTURE}

The LAR-RPTP subfamily is composed of three members in vertebrate organisms: PTPRF (also known as LAR), PTPRD (PTPS) and PTPRS (PTPo). These genes share up to $72 \%$ identity in humans (Coles et al., 2015). PTPRF, PTPRD and $P T P R S$ are located at chromosome 1 p34.2, chromosome 9 p24.1p23, and chromosome 19 p13.3, respectively, and as discussed in Section 3, all three LAR-RPTPs have multiple isoforms. LARRPTPs share a similar structure and domain organization, consisting of three extracellular Ig-like domains, eight extracellular fibronectin type III (FNIII) domains, a transmembrane region, and two intracellular protein tyrosine phosphatase (PTP) domains (Figure 1). In the extracellular 
region, the three Ig-like domains fold into a V-shaped conformation that is fundamental for LAR-RPTPs ligand binding activity (Kwon et al., 2010; Yamagata et al., 2015). The FNIII domains have a "beads on a string" conformation which is flexible for motility within the synaptic cleft, and to regulate clustering of LAR-RPTPs and their interaction with various ligands (Won and Kim, 2018).

The intracellular region of LAR-RPTPs consists of two PTP domains: a membrane proximal PTP domain (D1) that is catalytically active due to a cysteine residue that is required for substrate tyrosine dephosphorylation, and a membrane distal domain (D2) that has no catalytic activity but is important for stabilizing LAR-RPTPs in the synaptic zone (Chagnon et al., 2004). This function is fulfilled via binding to intracellular molecules, and by acting as a docking domain for the interaction with other receptors and scaffolding proteins such as liprins to modulate synapse formation (Streuli et al., 1990; Pulido et al., 1995; Dunah et al., 2005). Among the three LAR-RPTPs, D2 domains are more highly conserved than D1 (Krueger et al., 1990), and despite the lack of catalytic activity, in the case of PTPRF, replacement of two specific residues (Leu-1644-to-Tyr and Glu-1779-to-Asp) can restore phosphatase activity to the D2 domain (Nam et al., 1999). Therefore, the different domains of LAR-RPTPs confer them the particularity of acting as adhesion molecules and ligand receptors in the extracellular, and as phosphatases and scaffolding proteins in the intracellular, which illustrates the diversity of molecular functions of these proteins in the cell.

\section{LAR-RPTP ISOFORMS AND ALTERNATIVE SPLICING}

During neuronal development, alternative splicing events are precisely coordinated by the combinatorial effects of RNAbinding proteins, leading to neuron-specific splicing isoforms (Weyn-Vanhentenryck et al., 2018). In the case of LAR-RPTPs, alternative splicing regulates the inclusion of four mini-exons (short peptide sequences of up to 16 amino acids; meA-D, Figure 1). LAR-RPTPs mini-exon peptide sequences are encoded by micro-exons (nucleotide sequences of shorter than 30 nucleotides), which are part of a highly conserved and dynamic network where micro-exons are critical for neuronal alternative splicing events (Irimia et al., 2014; Li et al., 2015; Parada et al., 2021).

The inclusion of mini-exon meA is determined by the alternative splicing of two micro-exons, which yield three possible meA variants; meA3 (ESI), meA6 (GGTPIR) and their tandem combination meA9 (ESIGGTPIR), which are inserted in the Ig2 domain (Figure 1). The meB peptide sequence is encoded by a single micro-exon, which incorporates either a DQRE for PTPRF, or ELRE residues for PTPRS and PTPRD. In each case, the meB peptide is inserted between the Ig2 and Ig3 domains (Figure 1) (Yamagata et al., 2015; Yoshida et al., 2021). Mini-exons meC and meD are inserted in the FN5 domain and near the D1 catalytic domain respectively (Figure 1).

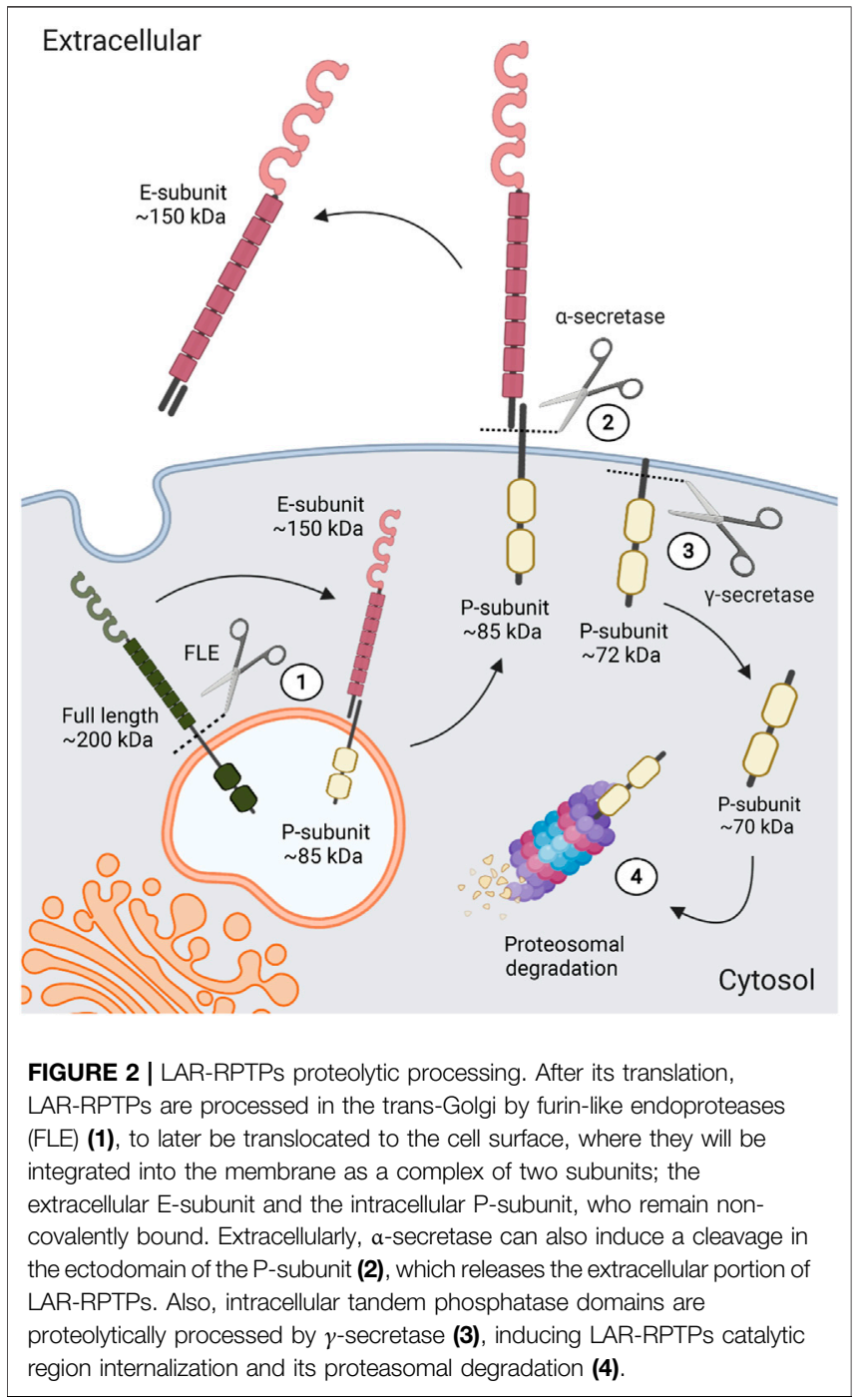

While the biological functions of $\mathrm{meC}$ and $\mathrm{meD}$ have not yet been described, meA and meB inclusions have fundamental roles in modulating LAR-RPTPs ligand binding activity. Therefore LAR-RPTPs alternative splicing contributes to a molecular code of the synaptic organization. Alternative splicing at meA and meB sites is key to determining the Ig-mediated binding affinities for a wide range of synaptic proteins, including TrkC, IL1RAPL1, IL1RAcP, Slitrks, SALMs, and Neurexins (Han et al., 2016; Han et al., 2020a, Li et al., 2015; Takahashi et al., 2011; Yoshida et al., 2011, 2012). For example, only the PTPRD isoforms that contain meA9 or meA6 can bind to IL1RAPL1. Although the meA6 variant requires the inclusion of meB to interact with IL1RAPL1, meA9 insertion alone is sufficient to enable PTPRD/IL1RAPL1 interaction (Yamagata et al., 2015). Also, since meA inserts on PTPRD only partially interact with IL1RAcP, the strength of PTPRD/IL-1RAcP interaction is mostly determined by meB (Han et al., 2016; Won and Kim, 2018). Although meB inclusion represents a subtle amino acid change of the LAR-RPTPs protein sequences, the functional consequences are profound since the $\mathrm{meB}$ insertion introduces a flexible linker 
between the Ig2 and Ig3 domains. In the case of PTPRD, the linker enables the interactions with IL-1RAcP, IL1RAPL1, and SALM3, and for PTPRS, it enables interaction with Slitrk and inhibits the interaction with TrkC (Han et al., 2016; Won and Kim, 2018).

The molecular mechanisms that regulate the expression of different LAR-RPTP isoforms are unknown. However, it can be hypothesized that a complex signaling network tightly regulates their alternative splicing, as it modulates LAR-RPTPs coupling to specific ligands and synaptic partners to drive fundamental neurodevelopmental processes such as synaptic differentiation (reviewed in Fukai and Yoshida, 2020; Han et al., 2016; Takahashi and Craig, 2013; Um and Ko, 2013). Therefore, the different LARRPTP isoforms generated by micro-exons alternative splicing might participate in different cellular processes, which highlights the importance of understanding the biological functions of each alternative splicing variant, especially those generated by the inclusion of $\mathrm{meD}$, since given its proximity to the catalytic domain, it could regulate substrate affinity of the LAR-RPTPs.

\section{PROCESSING AND REGULATION OF LAR-RPTPS ACTIVITY}

LAR-RPTPs are expressed as a $\sim 200-\mathrm{KDa}$ protein that can undergo proteolytic processing mediated by furin-like endoproteases in the trans-Golgi to be translocated to the cell surface (Figure 2) (Aicher et al., 1997; Serra-Pagès et al., 1994; Streuli et al., 1992). These endoproteases recognize a pentaarginine sequence at the C-terminus of the extracellular domain which generates a $150-\mathrm{KDa}$ extracellular subunit (E-subunit) that remains noncovalently bound to a $85-\mathrm{KDa}$ subunit (P-subunit) containing a short ectodomain, the transmembrane peptide, and the two intracellular phosphatase domains (Serra-Pagès et al., 1994). LAR-RPTPs can also undergo a second $\alpha$-secretase-dependent proteolytic processing at a site within the P-subunit ectodomain near the transmembrane region, which releases the extracellular region from the cell surface and promotes P-subunit internalization (Haapasalo et al., 2007). Mature LAR-RPTPs can also undergo proteolytic processing of the intracellular tandem phosphatase domains, which appears to play an important role in regulating LARRPTPs intracellular signaling. It has been observed that LARRPTPs catalytic activity is down-regulated by $\gamma$-secretase mediated proteolytic processing, which induces the internalization of the catalytic region for its proteasomal degradation, thereby reducing their intracellular signaling (Figure 2) (Aicher et al., 1997; Haapasalo et al., 2007; Coles et al., 2015). Alternatively, it has been proposed that the internalized LAR-RPTP catalytic region could regulate transcription, as PTPRF intracellular fragment cleaved by $\gamma$-secretase enter the nucleus and interact with $\beta$-catenin, dephosphorylating it and reducing its transcriptional activity (Haapasalo et al., 2007).

LAR-RPTPs cleaved E-subunit have been mostly observed in cell cultures media (Craig and Brady-Kalnay, 2011), suggesting that all three LAR-RPTPs are shed and might be exerting extracellular signaling through the released fragment. In the rat brain, it has been shown that a PTPRF short ectodomain (a segment of the fifth FNIII domain) forms a homophilic interaction with mature PTPRF to regulate neurite outgrowth (Yang et al., 2003). Similarly, PTPRD homophilic interaction with its extracellular fragment has been shown to promote axonal growth (Sun et al., 2000). Finally, although PTPRS homophilic interaction has not yet been documented, PTPRS ectodomains have been shown to promote neurite outgrowth non-cellautonomously (Sajnani, et al., 2005), suggesting that LARRPTPs cleaved extracellular domains act as paracrine signaling ligands that drive the growth of neuronal structures. More importantly, as LAR-RPTPs interact with several ligands to regulate neurobiological processes, cleaved ectodomains may antagonize mature LAR-RPTP interaction with their transsynaptic ligands. Therefore, extracellular proteolytic processing could be a negative-feedback mechanism for LAR-RPTPs transsynaptic signaling.

As mentioned before, the D2 domain lacks PTP activity as a result of a substitution of the critical residues that recognize the substrate phosphotyrosine, which initially suggested that the D2 domain played no role in catalysis (Streuli et al., 1990; Nam et al., 1999; Won and Kim, 2018). However, it has been shown that the D2 domain has an important function modulating D1 catalytic activity by regulating the substrate specificity (Streuli et al., 1990), and by participating in LAR-RPTP inhibition. It has been shown that heterodimerization of the PTPRD-D2 domain and the PTPRS-D1 domain negatively regulates catalytic activity of PTPRS without affecting the activity of PTPRD (Wallace et al., 1998). Also, it has been observed that LAR-RPTPs homophilic D1/D1 interaction decreases their phosphatase activity by hindering the substrate-binding pocket in the D1 domain (Coles et al., 2011; Xie et al., 2020), suggesting that LARRPTPs negatively regulate their phosphatase activity by forming dimers at the plasma membrane.

LAR-RPTPs homophilic or heterophilic interaction is mediated by a helix-loop-helix (HLH), wedge-shaped motif located between the membrane proximal region and the D1 domain, which mediates catalytic inhibition. The use of LARRPTP wedge peptides have been proved to successfully inhibit LAR-RPTPs functions in brain cells (Xie et al., 2006; Lang et al., 2015). Interestingly, in some cases LAR-RPTP wedge peptides can modulate their extracellular ligand binding activity. For example, in neuronal cell cultures, the treatment with peptidemimetics of the PTPRS wedge motif decreases the extracellular interaction of PTPRS with chondroitin sulfate proteoglycans (CSPGs) ligands (Lang et al., 2015), which indicates that LARRPTPs catalytic inhibition could induce conformational changes that modify ligand affinity.

It has also been observed that some LAR-RPTPs catalytic activity is modulated by oxidation, where the oxidative state of D1 and D2 domains determines LAR-RPTP phosphatase activity (Groen et al., 2005). PTPRS catalytic activity is reduced in cells exposed to UV through an unknown mechanism that oxidizes a cysteine in its active site in vitro (Groß et al., 1999). Also, it has been observed that oxidation induces PTPRF conformational changes in D1 and D2 domains, which promotes its 
dimerization (Groen et al., 2008), suggesting another mechanism for regulating LAR-RPTP catalytic activity. However, it remains to be determined how the microenvironment $(\mathrm{pH}$ and $\mathrm{UV}$ radiation) and the oxidative state of LAR-RPTPs modulates their catalytic activity.

LAR-RPTPs proteolytic processing appears to be important to regulate their signaling, although more studies are required to determine the physiological function of the different cleaved domains, especially in vivo. Also, their catalytic activity is tightly regulated by dimerization, which is mediated by the interaction between intracellular domains, suggesting an important role for LAR-RPTPs membrane clustering. However, the cellular mechanisms that promote dimerization of the LAR-RPTPs remain largely understudied, as well as the molecular changes induced by microenvironmental factors that modulate their catalytic activity.

\section{LAR-RPTPS IN NEURITE GROWTH AND AXON GUIDANCE}

In the last few decades, LAR-RPTPs have been shown to have important roles in several signaling pathways in the brain, such as in the regulation of dendrite and axon growth. LAR-RPTPs mediate cell-cell or cell-extracellular matrix adhesion to promote neurite outgrowth, and these processes often depends on the binding of LAR-RPTPs to CSPGs and heparan sulfate proteoglycans (HSPGs) (Johnson et al., 2006; Fisher et al., 2011; Chien and Ryu, 2013). The first in vitro demonstration that all three LAR-RPTPs participate in neurite outgrowth was carried out in retinal and hippocampal neurons, and PC12 cells (Tisi et al., 2000; Yang et al., 2003; Yang et al., 2005), which was confirmed later in studies using knockout mice and LAR-RPTPs blocking peptides (Fisher et al., 2011; Chien and Ryu, 2013). The intracellular pathways involved in axonal guidance have largely been described in invertebrate models such as Caenorhabditis elegans and Drosophila melanogaster. A major signaling effector is Trio, a guanine nucleotide exchange factor (GEF) for Rac1 and RhoA, which interacts with the D2 domain of LAR-RPTPs to promote axon guidance (Debant et al., 1996; Ball et al., 2010; Fuentes-Medel and Budnik, 2010). Trio also binds to ABL1 (also known as c-Abl) and Ena/VASP to regulate axon growth, in a mechanism that requires its direct interaction with Dlar, a Drosophila LAR-RPTP ortholog (Wills et al., 1999). The intracellular signaling mediated by Trio, ABL1 and Dlar is required for actin cytoskeleton remodeling associated with axon growth (Um and Ko, 2013). LAR-RPTPs also mediate motor axon guidance signaling via direct interaction with calcium/calmodulin-dependent serine protein kinaseinteracting proteins (Caskin 1 and Caskin 2), which form a signaling complex for axon growth and guidance (Weng et al., 2011).

\section{PTPRF}

A predominant function of PTPRF is the regulation of the actin cytoskeleton (Sarhan et al., 2016b). It has been observed that PTPRF knockout leads to a reduction in the number of focal adhesions and reduced adhesion to the extracellular matrix, suggesting PTPRF as a part of a complex that links actin cytoskeleton to the extracellular matrix and forms focal adhesions to promote neurite growth (Dunah et al., 2005; Sarhan et al., 2016a). PTPRF interaction with extracellular matrix ligands promote its catalytic activation and the dephosphorylation the tyrosine kinase ABL1, which promotes AKT and CDK1 activation (Sarhan et al., 2016a), increasing cell adhesion to extracellular matrix and favoring the growth of dendrites and axons (Serra-Pagès et al., 1995). Also, PTPRF homophilic interaction with its ectodomain has been shown to promote neurite outgrowth, in a mechanism where the ectodomain acts as a ligand to induce PTPRF phosphatase activity, which then participates in the intracellular activation of several signaling pathways (Yang et al., 2003, 2005).

\section{PTPRD}

A role for PTPRD in dendrite growth was discovered in PTPRD knockout mice, which display reduced dendritic branching, length, and thickness (Nakamura et al., 2017). PTPRD promotes dendrite growth by dephosphorylating and activating Fyn and Src kinases, which induces the arborization of dendrites mediated by Semaphorin-3A (Nakamura et al., 2017). Also, Semaphorin-3A-induced growth cone collapse response has been shown to be dependent on PTPRD expression, suggesting direct participation of PTPRD in axon growth (Nakamura et al., 2017). Besides, a soluble gradient of PTPRD induces chemoattraction of growth cones in neuronal cultures, in a mechanism dependent on tyrosine phosphatase activity (Sun et al., 2000), which highlights the dual role of PTPRD as a ligand and as a signaling molecule in axon growth regulation. However, PTPRD knockout mice do not show gross impairment in axon growth, while knocking out both PTPRD and PTPRS induces axon degeneration as peripheral nerves fail to contact their targets (Uetani et al., 2006). This indicates that LAR-RPTPs may have redundant roles in axon growth regulation (Stoker, 2015).

\section{PTPRS}

PTPRS has the opposite role in the development of dendrites compared to PTPRF and PTPRD, as PTPRS knockout mice show increased dendritic length in vivo (Horn et al., 2012). The inhibition of dendritic formation mediated by PTPRS depends on its direct interaction with its ligand CSPG, which induces PTPRS-mediated TrkB dephosphorylation, thereby suppressing dendritic spine growth (Figure 3A) (Kurihara and Yamashita, 2012; Lesnikova et al., 2020). Neurite outgrowth inhibition induced by CSPGs also appears to be mediated by the intracellular interaction between PTPRS and the nucleoside diphosphate kinase 2 NME2 (Figure 3A) (Hamasaki et al., 2016).

PTPRS also negatively regulates axon growth, as PTPRS knockout or catalytic inactivation has been widely reported to activate axonal elongation. Interaction with HSPGs ligands induces the formation of PTPRS dimers, which inactivates its phosphatase activity and promotes axon elongation (Figure 3B), while CSPGs engagement promotes the PTPRS monomer conformation, unleashing its catalytic activity and inhibiting 


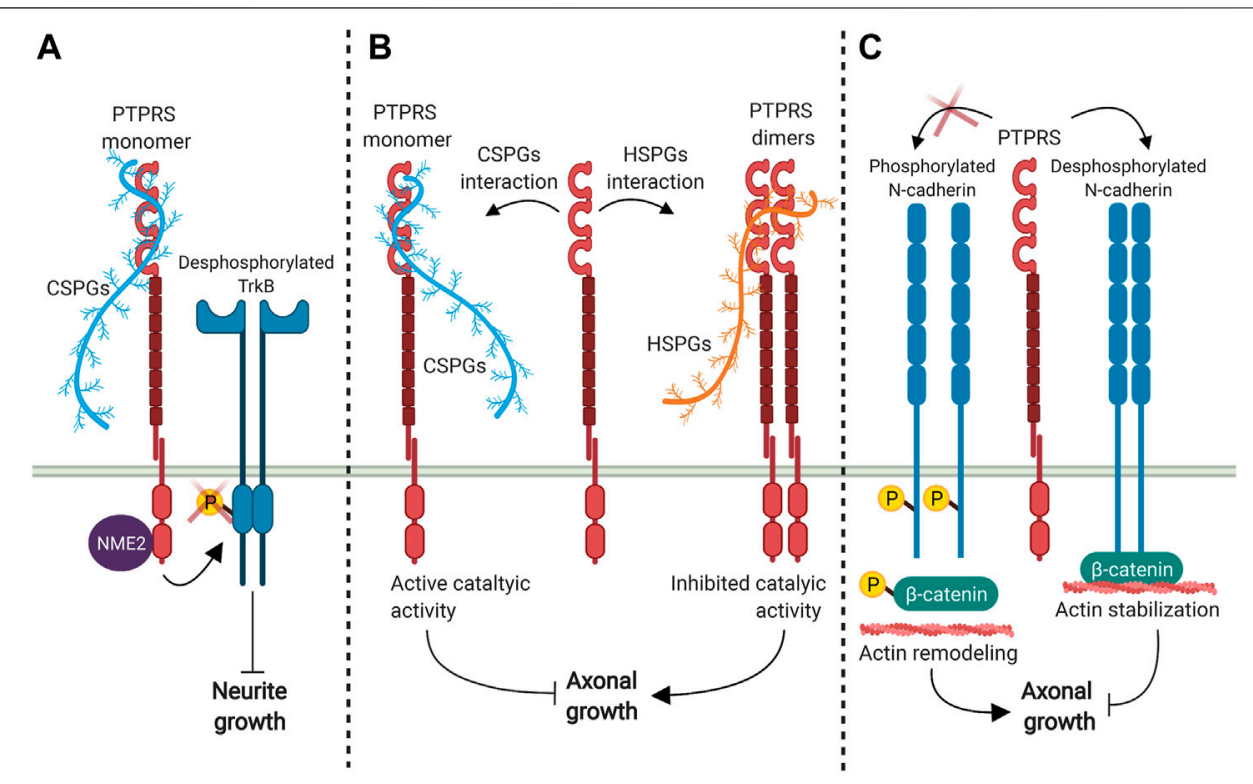

FIGURE 3|PTPRS signaling modulates dendrite and axon growth. (A) PTPRS interaction with CSPGs promotes TrkB dephosphorylation which reduces dendrite growth in a mechanism that appears to be mediated by PTPRS-NME2 interaction (Kurihara and Yamashita, 2012; Lesnikova et al., 2020). (B) PTPRS-HSPGs interaction induce PTPRS dimer formation, which inactivates its catalytic activity and favors axon growth; while the interaction with CSPGs promotes the PTPRS monomer conformation, inducing its catalytic activity and inhibits axonal growth (Shen et al., 2009; Coles et al., 2011). (C) PTPRS interaction with N-cadherin

dephosphorylates $\mathrm{N}$-cadherin and $\beta$-catenin, which favors $\mathrm{N}$-cadherin- $\beta$-catenin interaction, stabilizes actin cytoskeleton, and reduces axonal growth (Siu et al., 2007).

growth cone elongation (Coles et al., 2011; Lang, et al., 2015; Shen et al., 2009). Furthermore, embryonic cortical neurons isolated from PTPRS knockout animals showed an increased rate of axonal elongation (Thompson et al., 2003). PTPRS deficient animals also showed significantly accelerated axonal regeneration in facial motor neuron axotomy, sciatic nerve crush injury and spinal hemisection models (McLean et al., 2002; Thompson et al., 2003; Fry et al., 2009). The same results were observed when axonal regeneration was evaluated after optic nerve injury, where the number of axons that cross the lesion site was higher in PTPRS deficient mice (Sapieha et al., 2005). Besides, mice lacking PTPRS also show increased axon collateral branching in the hippocampus during normal aging or following chemically induced seizure (Horn et al., 2012), suggesting that PTPRS has an important role in maintaining neuronal structures by suppressing dendritic formation, and axonal growth and branching. Also, catalytic inhibition of PTPRS using a wedge motif peptide-mimetic efficiently restores axonal elongation in mice models of spinal cord injury, recovering the serotoninergic innervation into the spinal cord (Lang et al., 2015). Therefore, PTPRS may be a promising therapeutic target for axonal degeneration pathologies.

$\mathrm{N}$-cadherin and $\beta$-catenin have been proposed as the substrates that mediates PTPRS participation in the inhibition of axonal growth. The dephosphorylation of $\mathrm{N}$-cadherin and $\beta$-catenin by PTPRS promotes $\mathrm{N}$-cadherin- $\beta$-catenin complex formation, which favors the association between $\mathrm{N}$-cadherin and the actin cytoskeleton to reduce axonal growth (Figure 3C) (Siu et al., 2007). Another substrate proposed to interact with PTPRS is p250GAP (Chagnon et al., 2010), a GTPase-activating protein that regulate the small GTPases RhoA, Rac and Cdc42 (Moon and Zheng, 2003; Nakazawa et al., 2003). This protein is widely expressed in the embryonic and adult brain with an expression pattern similar to PTPRS (Schaapveld et al., 1998; Simó and Cooper, 2012). Neuronal cultures obtained from p250GAP deficient animals presented longer neurites compared to wild-type mice (Simó and Cooper, 2012), a phenotype similar to PTPRS-deficient neuronal cultures (Thompson et al., 2003), suggesting that p250GAP and PTPRS could be acting in the same pathway. As the activity of p250GAP increases in the presence of PTPRS (Chagnon et al., 2010), and its activity is inhibited by phosphorylation (Okabe et al., 2003), p250GAP dephosphorylation and activation mediated by PTPRS could therefore be important to restrict neurite outgrowth.

The participation of LAR-RPTPs in several signaling pathways that regulate neurite outgrowth and axon guidance reveals important roles of LAR-RPTPs to ensure correct brain development. However, a recent paper has disputed the participation of LAR-RPTPs in the regulation of neuronal morphology. Sclip and Südhof (2020) have found that knocking out LAR-RPTP expression after neurogenesis but before synapse formation do not affect dendritic and axonal growth, which was observed when the genes encoding all three LAR-RPTPs, singly or in combination, were deleted in cultured neurons, suggesting that LAR-RPTPs expression is expendable for neuronal development, at least in hippocampal neurons (Sclip and Südhof, 2020). These results, which contradict the evidence summarized above, suggest that functions of LAR-RPTPs in neuronal development needs to be revisited, and highlights the importance of studying the role of LAR-RPTPs in the brain 
considering factors such as cellular context and developmental stage (Tomita et al., 2020).

\section{LAR-RPTPS IN SYNAPTIC FORMATION AND FUNCTION}

The first evidence that suggested a role for LAR RPTPs in synapse formation came from their synaptic localization, which was demonstrated by immunofluorescence and coimmunoprecipitation with synaptic proteins such as $\beta$-catenin (Kypta et al., 1996; Wyszynski et al., 2002; Dunah et al., 2005; Um and Ko, 2013). $\beta$-catenin interacts with $\mathrm{N}$-cadherin to regulate dendritic spine morphogenesis and neurotransmitter vesicles release (Brigidi and Bamji, 2011; Um and Ko, 2013). As BDNF promotes synapse formation by inducing $\beta$-catenin phosphorylation, which reduces $\beta$-catenin-cadherin interaction (Bamji et al., 2006), it has been suggested that the direct dephosphorylation of $\beta$-catenin by LAR-RPTPs could downregulate synapse formation (Um and Ko, 2013). Although direct participation of LAR-RPTPs in $\beta$-catenin induced synaptic formation has not been demonstrated, there are numerous studies showing that LAR-RPTPs play fundamental roles in synapse formation though trans-synaptic interactions with key synaptic regulators such as SALM3, TrkC, Slitrks, NGL-3 and IL-1RAcP (Woo et al., 2009; Takahashi et al., 2011, 2012; Yoshida et al., 2012; Um et al., 2014; Li et al., 2015). As discussed later, these interactions often induce specific synaptic differentiation.

Recent literature has provided apparently conflicting observations regarding the role of LAR-RPTPs in synapse formation, probably due to the differential experimental approaches that have been carried out. While RNAi-mediated knockdowns of LAR-RPTPs in cell cultures have shown deficiencies in synaptic formation and neurotransmitter release (Dunah et al., 2005; Ko et al., 2015; Han et al., 2018), recent studies using conditional knockout animals have suggested that PTPRD and PTPRS are not essential for synaptic formation at least in the hippocampus (Han et al., 2020c; Sclip and Südhof, 2020). Despite the clear difference between both experimental models, it is important to highlight that other studies using LARRPTP knockout mice have shown impairments in behaviors such as spatial learning, memory, motor control and non-REM sleep as discussed later (Wallace et al., 1999; Uetani et al., 2000; Meathrel et al., 2002; Kolkman et al., 2004; Park et al., 2020). Therefore, the different phenotypes observed in constitutive knockout animals, versus mice lacking LAR-RPTPs in specific neural progenitor cells such as Nestin or Emxl expressing cells, could induce genetic compensation and a "masked" phenotype (Morrison and Münzberg, 2012; Luo et al., 2020). Additional studies using other Cre-driver genes should be evaluated to determine the different cellular mechanisms impaired in each animal model.

Even though the three LAR-RPTPs are mainly expressed presynaptically, where they participate in synaptic differentiation, their expression has been observed postsynaptically at excitatory synapses (Wyszynski et al., 2002; Dunah et al., 2005). It has been suggested that postsynaptic LARRPTPs participate in receptor trafficking, reducing the density of
AMPA receptors (AMPAR) in hippocampal synapse (Ko et al., 2003a; Dunah et al., 2005; Brigidi and Bamji, 2011). Likewise, LAR-RPTPs regulate AMPAR synaptic transmission through a mechanism mediated by LAR-RPTPs and SALM5 interaction, which promotes the dephosphorylation of AMPAR subunits (Choi et al., 2016). This induces AMPAR internalization and promotes long term depression (LTD) (Dunah et al., 2005; Dickinson et al., 2009). Also, it has been observed that all three LAR-RPTPs control synapse properties by regulating NMDAR-mediated responses, and thus have a critical role in synaptic transmission (Sclip and Südhof, 2020).

\section{PTPRF}

Presynaptic PTPRF participates indirectly in LTD through its interaction with netrin-G ligand-3 (NGL-3) when promotes synaptic differentiation (Woo et al., 2009; Kwon et al., 2010). Treatment with NMDA in cultured neurons or lowfrequency stimulation in brain slices induces the proteolytic cleavage of NGL-3, which disrupts the trans-synaptic interaction between NGL-3 and PTPRF, impairing synaptic adhesion during LTD, and weakening excitatory synapses (Lee et al., 2014).

\section{PTPRD}

PTPRD knockout mice show enhanced long-term potentiation (LTP) in hippocampal synapses, possibly due to increased neurotransmitter release in the CA1 region. This induces behavioral alterations such as impaired spatial learning and memory, and motor deficits (Uetani et al., 2000), illustrating the importance of PTPRD for hippocampal LTP formation. However, it has also been shown that PTPRD knockout mice have impaired locomotive behaviors and motor weakness, as well as a transient delay in myelination at early postnatal development (Drgonova and Walther, 2015; Zhu et al., 2015), which could partially explain their altered behavior observed in learning tests. PTPRD knockout mice also have impaired synaptic development and decreased excitatory synaptic transmission mainly due to the dysfunction in its interaction with IL1RAPL1 (Park et al., 2020), which highlights the role of PTPRD not only in synaptic formation, but also in excitatory neurotransmission. In contrast, a recent study using pan-neuronal PTPRD conditional knockout animals, showed that PTPRD is not essential for maintenance of excitatory or inhibitory synaptic transmission, since absence of PTPRD did not modify synaptic parameters such as the number of excitatory or inhibitory synapses, miniature excitatory postsynaptic currents (mEPSC), synaptic vesicle tethering, active zone modifications nor neurotransmitter release (Han et al., 2020c), suggesting that PTPRD does not play a significant role in these neurobiological processes. It is important to highlight that Park et al. (2020) found that interaction between PTPRD and IL1RAPL1 is dependent on PTPRD alternative splicing. As much of the signaling mediated by PTPRD depends on interaction with specific ligands, different alternative splicing variants might participate in different cellular processes. Therefore, the controversial 
results observed in recent studies should be analyzed cautiously, as different alternatively spliced isoforms of PTPRD could generate different neuronal outcomes.

\section{PTPRS}

In presynaptic terminals, PTPRS has been shown to participate in excitatory synaptic function by regulating the localization and size of excitatory synaptic vesicles, modulating glutamate release in the hippocampus, and it also seems to regulate structural features of the active zone such as its length, suggesting that PTPRS participates in the molecular organization of the active zone machinery to efficiently promote glutamate release (Han et al., 2020b). PTPRS is also implicated in regulation of the postsynaptic excitatory neurotransmission. PTPRS interacts with GPC-4 and LRRTM4 to regulate frequency and amplitude of excitatory synaptic transmission. Studies in cultured hippocampal neurons showed that PTPRS knockdown decreased the frequency and amplitude of mEPSC, an effect that was reversed by re-expression of wild-type PTPRS, but not by heparan sulfate (HS)-binding-defective PTPRS mutant that impairs its interaction with GPC-4 and LRRTM4. These observations suggest that PTPRS-GPC-4-LRRTM4 interaction has an important role in the maintenance and function of excitatory synapses (Ko et al., 2015). However, these results contradict previous findings, where mice lacking PTPRS showed increased frequency of mEPSC, which resulted in reduced LTP, and greater paired-pulse facilitation (Horn et al., 2012). One possible explanation could be differential roles for PTPRS splice variants. As in the case of PTPRD, the different synaptic functions in which PTPRS participates must be carefully studied considering the different splicing isoforms, as the interaction of PTPRS with its ligands also depends on alternative splicing (Han et al., 2020a). Recent studies have proposed possible mechanisms by which PTPRS regulates excitatory synapses in the hippocampus (Kim et al., 2020; Sclip and Südhof, 2020). These studies strongly suggest that presynaptic PTPRS promotes LTP through its regulation of NMDAR in the postsynaptic. This is supported by results showing that postsynaptic deletion of PTPRS or the deletion of its extracellular regions required for trans-synaptic adhesions do not affect LTP, and therefore PTPRS ligand binding activity would be expendable for NMDAR regulation (Kim et al., 2020). It was also observed that PTPRS catalytic activity mediates dephosphorylation of presynaptic Neurexin-1, which interacts with PTPRS though a complex with liprin- $\alpha$ among others (Serra-Pagès et al., 1995; Hata et al., 1996; Olsen et al., 2005; Weng et al., 2011; Bomkamp et al., 2019), to promote NMDARmediated postsynaptic activity (Dai et al., 2019; Kim et al., 2020). These results suggest a mechanism mediated by PTPRS-neurexin-1 presynaptic intracellular complex that regulates NMDAR postsynaptic activation and LTP induction, which is important for recognition memory and novelty preference (Kim et al., 2020). This finding correlates with results obtained by Sclip and Südhof (2020), where a triple conditional knockout mouse for all three LAR-RPTPs show a decrease in NMDAR-mEPSCs in the hippocampus, which is due to a reduction in NMDAR postsynaptic location and not to NMDAR protein levels (Sclip and Südhof, 2020).
The evidence summarized here shows that LAR-RPTP interactions with trans-synaptic ligands and with their intracellular substrates modulates a series of synaptic processes required for neuronal function. Even though recent papers have questioned LAR-RPTP participation in some of these biological processes, several evidence showing a primary role in synaptic formation suggest that LAR-RPTP functions can not be underestimated, and more studies are needed to unravel the differential roles that LAR-RPTP alternative splicing variants might have over these neuronal functions.

\section{LAR-RPTPS IN SYNAPSE DIFFERENTIATION}

It is well known that the main roles of LAR-RPTPs at the synapse are as adhesion molecules and as mediators of synapse differentiation. LAR-RPTPs induce presynaptic differentiation by participating in recruitment of synaptic vesicles and the release/recycling machinery, and in some cases, they can also induce postsynaptic differentiation by recruiting neurotransmitter receptors and scaffolding and signaling proteins (reviewed in Takahashi and Craig, 2013). LARRPTPs are often located presynaptically at the axon, where they modulate excitatory or inhibitory differentiation depending on their trans-synaptic interaction partner (Figure 4). The major trans-synaptic partners for each LAR-RPTP have been described, and their interaction determines whether the synapse will be excitatory or inhibitory (Fukai and Yoshida, 2020; Han et al., 2016; Takahashi and Craig, 2013; Um and Ko, 2013). Also, LARRPTP interaction with specific partners can shape synaptic differentiation either in a unidirectional manner, inducing differentiation pre-synaptically only, or in a bidirectional way, where this interaction induces differentiation both presynaptically and post-synaptically (Figure 4). The mechanisms have not been completely characterized in mammals, but apparently presynaptic differentiation is mediated by the scaffolding proteins liprin-a (Serra-Pagès et al., 1998; Xie et al., 2020). Liprin-a proteins participate in synaptic scaffolding and differentiation (Spangler and Hoogenraad, 2007), and they were the first intracellular proteins shown to interact with D1 and D2 domains of LARRPTPs through their C-terminal SAM (sterile $\alpha$-motif) domains (Pulido et al., 1995; Spangler and Hoogenraad, 2007; Xie et al., 2020). Liprin interaction with D1 and D2 domains of LARRPTPs promotes presynaptic differentiation (Kaufmann et al., 2002; Dunah et al., 2005; Han et al., 2018), possibly through liprin-a coupling with synaptic proteins such as RIM1 $\alpha$ and ELKS/ERC, which integrates a molecular scaffold in the active zone that mediates neurotransmitter release (Schoch et al., 2002; Ko et al., 2003b). Furthermore, liprin-a regulates the location of LAR-RPTPs by promoting membrane clustering, which also inhibits LAR-RPTPs catalytic activity as mentioned previously (Serra-Pagès et al., 1998; Bomkamp et al., 2019; Xie et al., 2020). 


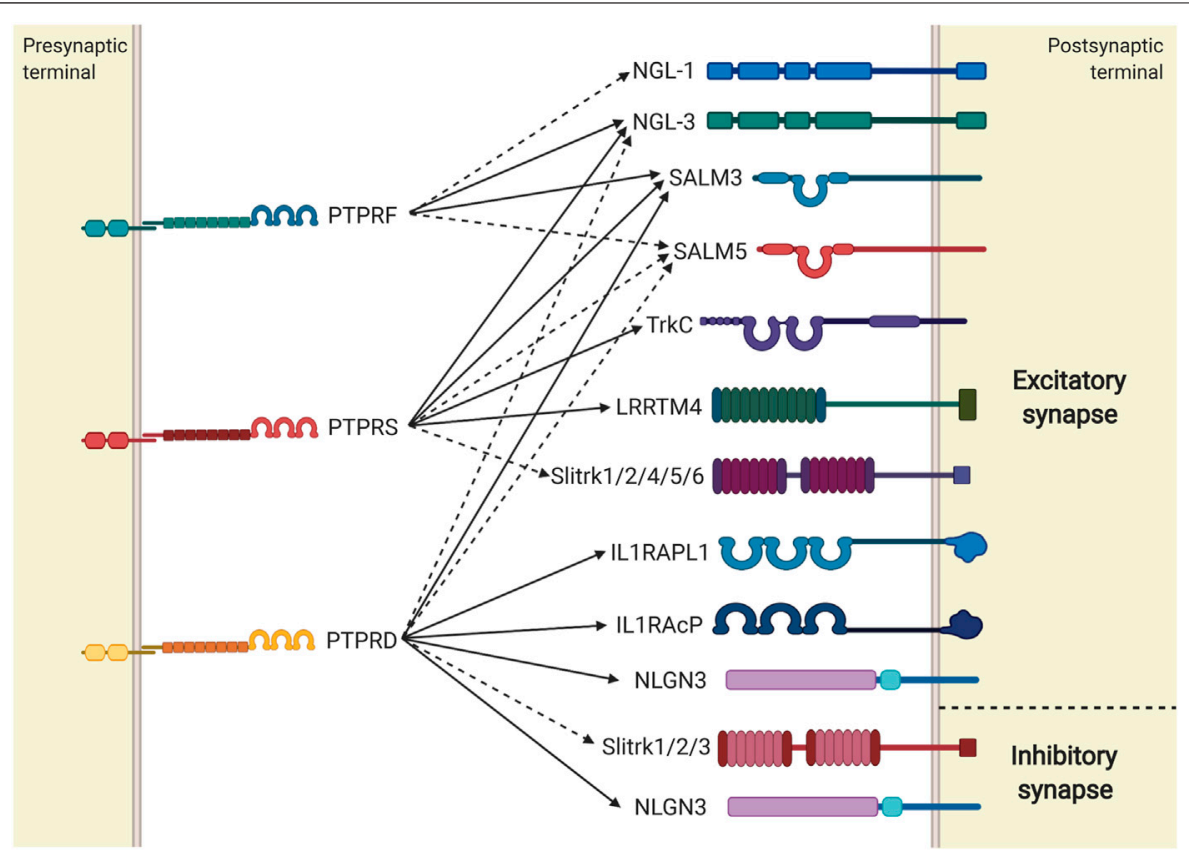

FIGURE 4 | LAR-RPTPs trans-synaptic interactions induce synaptic differentiation. Summary of LAR-RPTPs and their synaptic partners whose interactions induce excitatory or inhibitory synapse differentiation. LAR-RPTPs interactions that induce differentiation unidirectionally are represented with dashed lines, while interactions inducing bidirectional differentiation are represented with solid lines.

\section{Synaptic Partners Common for All LAR-RPTPs}

The first molecule discovered to interact trans-synaptically with all three LAR-RPTPs was NGL-3, a synaptic adhesion molecule involved in synaptic formation and neurotransmission (Kwon et al., 2010; Woo et al., 2009). Interaction between PTPRF and PTPRS with NGL-3 induces pre and postsynaptic differentiation when contacting axons and dendrites respectively, forming a trans-synaptic complex that induces bidirectional excitatory synaptic formation (Kwon et al., 2010; Woo et al., 2009). Presynaptic PTPRF and PTPRS interact with postsynaptic NGL-3 to promote excitatory postsynaptic differentiation, which is mediated by the direct interaction between NGL-3 and PSD-95 (Kwon et al., 2010). PTPRD also interacts with NGL-3 to promote excitatory differentiation, but in a unidirectional manner. Therefore, PTPRD appears to be the only LAR-RPTP unable to induce PSD-95 recruitment and postsynaptic differentiation when interacting with NGL-3 (Kwon et al., 2010). All three LAR-RPTPs also interact with SALM3 and SALM5, members of the SALM family of cell adhesion-like proteins that modulate differentiation, maintenance, and plasticity of the synapse (Ko et al., 2006), and their interaction with LAR-RPTPs promotes excitatory synapse development (Choi et al., 2016; Li et al., 2015; Lie et al., 2016). The interaction between LAR-RPTPs and SALM3 synaptic differentiation is apparently induced in a bidirectional manner, since SALM3 binding to each LAR-RPTP recruits excitatory presynaptic proteins (Li et al., 2015), and aggregation of SALM3 on dendritic surfaces induces clustering of PSD-95 (Mah et al., 2010). Interaction between SALM3 and LAR-RPTPs can be inhibited by the cis-interaction of SALM3 and SALM4, and therefore this interaction induces an inhibition of the SALM3-dependent excitatory presynaptic differentiation (Lie et al., 2016). On the other hand, the interaction between LARRPTPs and SALM5 induces unidirectional presynaptic differentiation due to lack of a PDZ-binding motif necessary to interact with PSD-95 in the postsynaptic (Choi et al., 2016; Goto-Ito et al., 2018; Mah et al., 2010).

\section{PTPRF Synaptic Partners}

Besides NGL-3 and SALM3/5, a PTPRF cis-interaction with netrin-G1 has been observed, which can also induce excitatory differentiation in a unidirectional manner. Presynaptic netrin-G1 interacts with postsynaptic netrin-G ligand-1 (NGL-1), and simultaneously directly interacts with adjacent PTPRF to shape pre-synaptic excitatory synapsis (Song et al., 2013). However, it remains to be confirmed if this interaction also occurs in vivo.

\section{PTPRD Synaptic Partners}

PTPRD is the only LAR-RPTP whose expression has been observed in both excitatory and inhibitory synapses, where its interaction with NGL-3, SALM3/5, IL1RAPL1 and IL1RAcP induce excitatory differentiation, while its interaction with Slitrk1, Slitrk2 and Slitrk3 induce inhibitory synaptic differentiation (Goto-Ito et al., 2018; Kwon et al., 2010; Li et al., 2015; Lin et al., 2018; Park et al., 2020; Takahashi et al., 2012; Valnegri et al., 2011; Yim et al., 2013; Yoshida et al., 2011; Yoshida et al., 2012). Axonal PTPRD induces excitatory synaptic 
development bidirectionally through the interaction with its postsynaptic ligand IL1RAPL1 (Valnegri et al., 2011; Yoshida et al., 2011; Park et al., 2020). PTPRD-IL1RAPL1 interaction recruits RhoGTPase-activating protein 2 (RhoGAP2) in the postsynaptic density which promotes a signaling pathway that favors excitatory synapse development and dendritic spine formation (Valnegri et al., 2011; Park et al., 2020). Also, the postsynaptic excitatory differentiation induced by the interaction of PTPRD and IL1RAPL1 depend on the modulation of c-Jun terminal kinase (JNK) signaling pathway, since PTPRD activates IL1RAPL1, inducing JNK activation, which phosphorylates PSD-95 and promotes its synaptic clustering (Pavlowsky et al., 2010; Park et al., 2020). However, whether RhoGAP2 participates in JNK-mediated synaptic differentiation has not been clarified. Also, PTPRD interacts with a brain isoform of IL1RAcP, a protein essential for immune response, which promotes excitatory synaptic differentiation bidirectionally (Yoshida et al., 2012; Yamagata et al., 2015). Although IL1RAcP has homology to IL1RAPL1, it is not known whether they share a common mechanism for PTPRD-mediated synaptic differentiation. Slitrk $1 / 2 / 3$ are also synaptic partners for PTPRD, and their interaction induces inhibitory synapse development in a unidirectional manner, since the complex induces a presynaptic GABAergic synapse differentiation, without necessarily shaping the postsynaptic (Takahashi et al., 2012; Yim et al., 2013).

Recently, a study has identified Neuroligin3 (NLGN3) as a new ligand for PTPRD. This interaction induces excitatory or inhibitory post-synaptic differentiation depending on microexon meB inclusion (Yoshida et al., 2021). Furthermore, it was also observed that PTPRD and NLGN3 mediate social behaviors such as social preference and negative social response and regulate excitatory/inhibitory synaptic differentiation (Yoshida et al., 2021), highlighting the role of PTPRD splicing and its isoform interactions in excitatory/inhibitory balance.

\section{PTPRS Synaptic Partners}

TrkC is one of the major postsynaptic partners for PTPRS, and their trans-synaptic interaction generates the development of excitatory synapse in a bidirectional manner (Takahashi et al., 2011; Han et al., 2018), where this complex recruits synapsin (mediated by the D2 domain of PTPRS) in the presynaptic and PSD-95 at the postsynaptic (Takahashi et al., 2011). Also, TrkC competes with HS for PTPRS interaction. HS-bound PTPRS molecules tend to assemble as oligomers at the presynaptic membrane, which inactivates its catalytic activity and promotes axon growth, whilst unbound PTPRS monomers tend to interact with TrkC to induce synaptic differentiation (Coles et al., 2011; Won et al., 2017; Han et al., 2018; Han et al., 2020a). Therefore, PTPRS interaction with both ligands and its consequent participation in each cellular process must be tightly regulated, suggesting the existence of an undefined developmental mechanism to switch PTPRS functions. When oligomeric PTPRS interacts with HS, it forms a cis-complexes with glypican-4 (GPC-4) with high affinity (Ko et al., 2015). GPC4 also interacts with the postsynaptic molecule LRRTM4, which induces a bidirectional excitatory synaptic differentiation mediated by PTPRS-GPC-4-LRRTM4 complex formation, and where presynaptic differentiation depends on the PTPRS catalytic activation induced by GPC-4 binding (Ko et al., 2015; Roppongi et al., 2020). This mechanism is developmentally regulated, since PTPRS only interacts with cleaved GPC-4, and its proteolytic cleavage is reduced during postnatal development (Ko et al., 2015). On the other hand, it has been observed that LRRTM4mediated synaptic differentiation is also dependent on PTPRS cis interaction with HS chains of Neurexins, where PTPRSNeurexin-LRRTM4 and PTPRS-GPC-4-LRRTM4 complexes would coexist independently to induce the same process (Roppongi et al., 2020). However, a recent study has suggested that PTPRS cis interaction with HS chains of Neurexins would have an antagonistic role in synaptic differentiation. In mouse cultured hippocampal neurons, the interaction between PTPRS and HS chains of Neurexin $1 \alpha$ inhibited excitatory post-synaptic differentiation (Han et al., 2020a). It is important to highlight that HS binding to PTPRS is mediated through Ig like domains and its affinity is highly dependent on PTPRS alternative splicing (Han et al., 2020a). Therefore, synaptic differentiation mediated by PTPRS should be carefully addressed since PTPRS splice variants might modulate differential synaptic pathways even when interacting with the same ligand. Presynaptic PTPRS interacts with Slitrks 1, 2, 4, 5 and 6 through the Ig domains to coordinate the development of the excitatory synapse in a unidirectional manner (Yim et al., 2013; Han et al., 2018). Slitrks-mediated presynaptic differentiation depends on the direct interaction between liprin- $\alpha$ and the PTPRS D2 domain, and also on the recruitment of the presynaptic proteins p250GAP and $\mathrm{N}$-cadherin (Han et al., 2018), showing that PTPRS binding to Slitrks ligands promotes the intracellular assembly of a complex presynaptic differentiation machinery.

Since the interaction of LAR-RPTPs with their synaptic partners controls the formation of excitatory and inhibitory synapses, with profound consequences for the development of the brain, their expression must be regulated in a highly coordinated manner during neurodevelopment. Although the mechanisms involved in the cell-specific expression of LARRPTPs and their synaptic partners are unknown, it is possible to suggest that those neurodevelopmental disorders in which the balance between excitatory and inhibitory synapses is lost may be due at least in part to dysregulations in the mechanisms that control the expression of LAR-RPTPs (Nelson and Valakh, 2015; Parenti et al., 2020; Yoshida et al., 2021).

\section{LAR-RPTPS IN BRAIN DEVELOPMENT}

Studies using LAR-RPTP knockout mice have revealed the importance of these molecules in brain development. Mice lacking the expression of each LAR-RPTP show neurodevelopmental phenotypes such as alteration in the number and size of neurons and neural precursors, altered neurogenesis in the hippocampus and brain cortex, aberrant cytoarchitecture, enhanced axon sprouting and axon targeting defects (Bernabeu et al., 2006; Takahashi and Craig, 2013; Um and Ko, 2013; Tomita et al., 2020). Accordingly, LAR-RPTPs are 


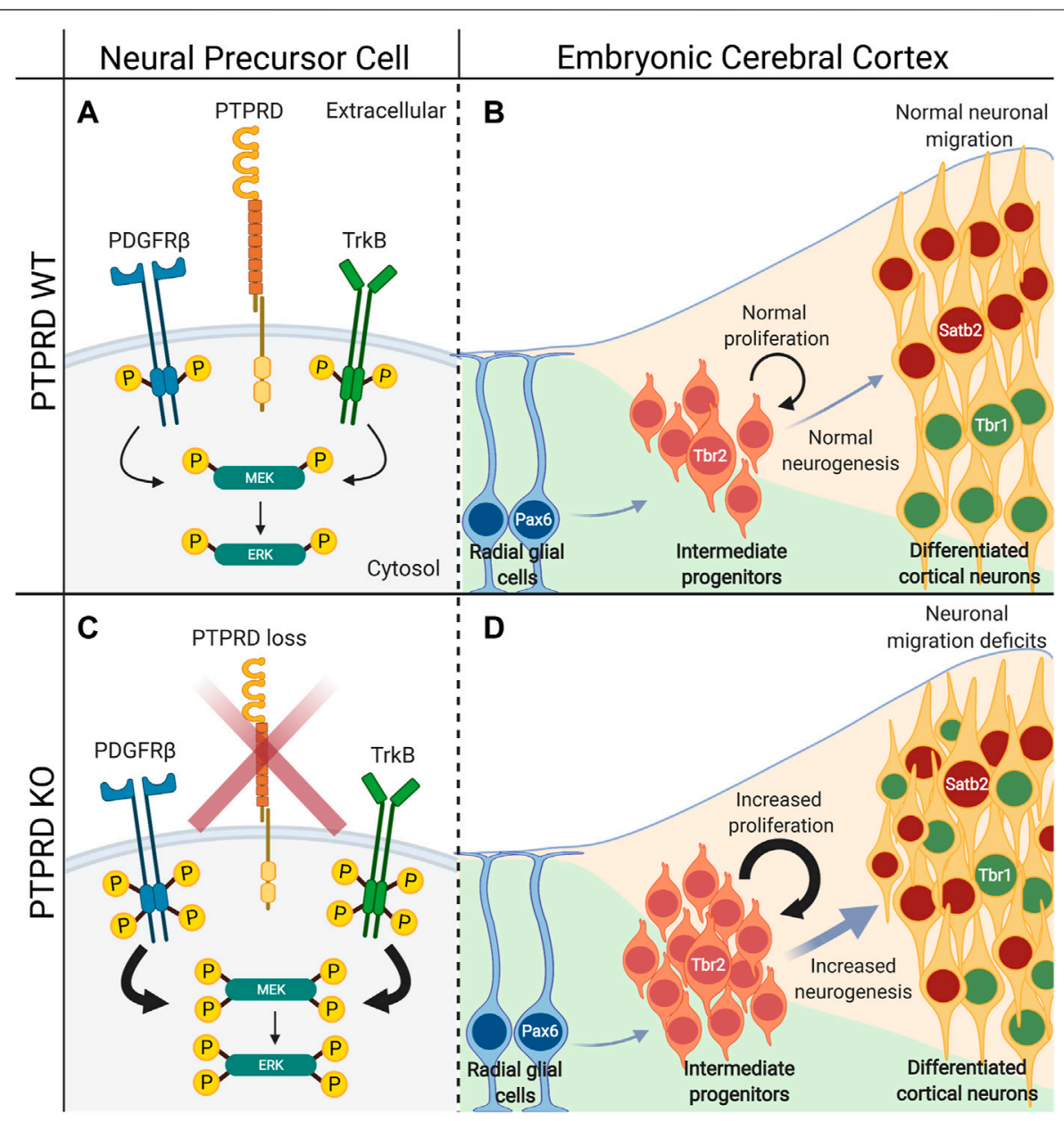

FIGURE 5 | PTPRD absence induce aberrant embryonic cortical neurogenesis. (A) PTPRD dephosphorylates PDGFR $\beta$ and TrkB receptor tyrosine kinases to control their activity, and the activation of MEK/ERK intracellular signaling. (B) This induces the normal Tbr2-positive intermediate progenitor cells proliferation and neurogenesis, and the correct localization of Satb2 and Tbr1-positive neurons into the brain cortex. However (C) when PTPRD expression is lost, NPCs have increased phosphorylation of PDGFR $\beta$ and TrkB, which derives in the hyperactivation of the MEK/ERK intracellular signaling. (D) This induces an increase in Tbr2-positive intermediate progenitor cell proliferation, and consequently, aberrant increased neurogenesis and impaired positioning of Satb2 and Tbr1-positive neurons into the brain cortex.

also implicated in developmental disorders through mechanisms such as reductions in intrinsic programmed cell death and stem cell proliferation (Uetani et al., 2009; Stewart et al., 2013).

\section{PTPRF}

PTPRF knockout mice display increased proliferation in embryonic hippocampal neural precursor cells (NPCs) in vitro and in adult hippocampal NPCs in vivo, which is associated with an increased hippocampal neurogenesis in the adult mouse brain (Bernabeu et al., 2006), although the mechanism for PTPRF neurogenic regulation has not yet been characterized.

\section{PTPRD}

Recently, our group found that PTPRD knockout mice have impaired neuronal differentiation and neuronal localization in the brain cortex (Tomita et al., 2020). First, we observed that the PTPRD heterozygous or homozygous knockout mice display increased neuronal intermediate progenitor cells (Tbr2 positive) without changing radial glial cell numbers (Pax6 positive). Also, the intermediate progenitor cells are highly proliferative, resulting in an increased number of differentiated cortical neurons and mislocalization of Satb2 and Tbr1-positive neurons into their corresponding cortical layers. These effects seem to be dependent on PTPRD catalytic activity and its interaction with the neurogenesis-associated receptor tyrosine kinases TrkB and PDGFR $\beta$. Indeed, PTPRD deletion increases phosphorylation of both receptors and downstream kinase effectors MEK1 and ERK1/2 (Figure 5). Furthermore, in vitro inhibition of this signaling pathway by either pharmacological or RNAi strategies rescues neurogenic impairments observed in the absence of PTPRD (Tomita et al., 2020), providing a direct link between PTPRD mutations and neurodevelopmental disorders. However, it is important to highlight that considering differential expression of PTPRD in a variety of neural cell populations and elucidating how PTPRD controls receptor tyrosine kinases will be key for determining the mechanisms by which PTPRD regulates 
TABLE 1 | LAR-RPTPs knock out models and their phenotypes. Different animal models lacking LAR-RPTPs expression and its induced phenotype have been summarized.

\begin{tabular}{|c|c|c|c|c|}
\hline $\begin{array}{l}\text { LAR- } \\
\text { RPTP(s) }\end{array}$ & Model & Cellular phenotype & Behavioral impairments & References \\
\hline PTPRF & PTPRF KO & $\begin{array}{l}\downarrow \text { focal adhesions, adhesion to ECM and } \\
\text { neurite growth } \\
\uparrow \text { NPCs proliferation and neurogenesis in } \\
\text { the hippocampus } \\
\downarrow \text { number and size of cholinergic neurons } \\
\downarrow \text { hippocampal cholinergic innervation } \\
\downarrow \text { regeneration and collateral axonal } \\
\text { sprouting }\end{array}$ & $\begin{array}{l}\text { Spatial learning impairments. } \\
\text { Increased nocturnal activity }\end{array}$ & $\begin{array}{l}\text { Bernabeu et al. (2006), Dunah et al. (2005), Kolkman } \\
\text { et al. (2004), Sarhan et al. (2016b), Van der Zee et al. } \\
\text { (2003), Van Lieshout et al. (2001), Yeo et al. (1997) }\end{array}$ \\
\hline \multirow[t]{3}{*}{ PTPRD } & PTPRD KO & $\begin{array}{l}\downarrow \text { dendritic branching, length, and } \\
\text { thickness } \\
\uparrow \text { axon degeneration } \\
\uparrow \text { hippocampal LTP } \\
\uparrow \text { cortical neurogenesis } \\
\uparrow \text { neuronal differentiation } \\
\downarrow \text { cortical neuronal migration }\end{array}$ & $\begin{array}{l}\text { Impaired spatial learning } \\
\text { Impaired memory } \\
\text { Impaired locomotive behaviors } \\
\text { Motor deficits }\end{array}$ & $\begin{array}{l}\text { Drgonova and Walther (2015), Nakamura et al. (2017), } \\
\text { Tomita et al. (2020), Uetani et al. (2000), Uetani et al. } \\
\text { (2006), Zhu et al. (2015) }\end{array}$ \\
\hline & $\begin{array}{l}\text { PTPRD cKO } \\
\text { (Emx1-Cre) }\end{array}$ & $\begin{array}{l}\downarrow \text { synaptic development } \\
\downarrow \text { excitatory synaptic transmission }\end{array}$ & $\begin{array}{l}\text { Hyperactivity } \\
\text { REM sleep disturbances }\end{array}$ & Park et al. (2020) \\
\hline & $\begin{array}{l}\text { PTPRD cKO } \\
\text { (Nestin-Cre) }\end{array}$ & $\begin{array}{l}\text { Normal number of excitatory and } \\
\text { inhibitory synapses } \\
\text { Normal synaptic transmission } \\
\text { Normal vesicle tethering } \\
\text { Normal neurotransmitters release at } \\
\text { postsynaptic targets }\end{array}$ & & Han et al. (2020c) \\
\hline \multirow[t]{2}{*}{ PTPRS } & PTPRS KO & $\begin{array}{l}\uparrow \text { dendritic density and length } \\
\uparrow \text { axon growth } \\
\uparrow \text { axonal elongation rate } \\
\uparrow \text { growth cone elongation } \\
\uparrow \text { axon regeneration } \\
\uparrow \text { axon collateral branching } \\
\uparrow \text { mEPSC frequency and paired-pulse } \\
\text { facilitation } \\
\downarrow \text { LTP in the hippocampus } \\
\uparrow \text { NPCs migration } \\
\downarrow \text { ChAT-positive neurons } \\
\downarrow \text { myelination } \\
\downarrow \text { luteinizing hormone-releasing cells }\end{array}$ & $\begin{array}{l}\text { Increased recognition memory. } \\
\text { Spastic movements, tremor and } \\
\text { ataxic gait. } \\
\text { Abnormal limb flexion. } \\
\text { Defective proprioception }\end{array}$ & $\begin{array}{l}\text { Coles et al. (2011), Elchebly et al. (1999), Fry et al. } \\
\text { (2009), Horn et al. (2012), Kirkham et al. (2006), Lang } \\
\text { et al. (2015), Meathrel et al. (2002), McLean et al. } \\
\text { (2002), Shen et al. (2009), Sapieha et al. (2005), } \\
\text { Thompson et al. (2003), Wallace et al. (1999) }\end{array}$ \\
\hline & $\begin{array}{l}\text { PTPRS cKO } \\
\text { (Emx1-Cre) }\end{array}$ & $\begin{array}{l}\downarrow \text { NMDAR-dependent synaptic } \\
\text { transmission and plasticity in the } \\
\text { hippocampus }\end{array}$ & $\begin{array}{l}\text { Deficits recognition memory } \\
\text { Impairment in social novelty }\end{array}$ & Kim et al. (2020) \\
\hline
\end{tabular}

these biological processes during embryonic cortical development.

\section{PTPRS}

In the spinal cord, it has been observed that PTPRF and PTPRS interaction with CSPGs inhibits NPC growth, attachment, survival, proliferation, and oligodendrocyte differentiation from NPCs (Dyck et al., 2015). Although the participation of PTPRF and PTPRS in NPC biology and neurodevelopment have not yet been characterized in the brain, NPCs cultured from the subventricular zone of PTPRS knockout mice show increased cellular migration from the neurosphere center, (Kirkham et al., 2006), suggesting a role of PTPRS in controlling NPC migration. In addition, mice lacking PTPRS expression show several neurological defects such as reduced number of choline acetyl transferase (ChAT)-positive neurons, slower nerve conduction velocity as a consequence of smaller myelinated fibers and hypomyelination, accompanied by behavioral alterations such as spastic movements, tremor and abnormal limb flexion among others. This suggests a role for PTPRS in the differentiation and/ or development of cholinergic neurons and glial cells (Wallace et al., 1999). In addition, these mice showed deficits in the formation of the pituitary, with an elongated intermediate lobe, and smaller anterior and posterior lobes. This is accompanied by an overall decrease in brain size, a smaller olfactory bulb, and a severe depletion of luteinizing hormonereleasing-positive cells associated to a reduced size of the hypothalamus (Elchebly et al., 1999), suggesting a role for PTPRS in the development of neural cells in some brain areas including the hypothalamus-pituitary axis.

The evidence summarized in this section shows that LARRPTPs participate in the regulation of NPCs proliferation, neural 
differentiation, and neuronal migration, showing an important role in brain development, and their potential participation in the etiology of some neurodevelopmental disorders due to their impaired expression. However, the role of LAR-RPTPs in neurodevelopment remains an understudied field since LARRPTP ligands and substrates that participate in brain development have not been fully characterized.

\section{ROLE OF LAR-RPTPS IN NEUROLOGICAL DISORDERS}

Many of the pathologies associated with LAR-RPTPs are systemic dysfunctions including cancer, metabolic diseases, and ulcerative colitis, among others (Chagnon et al., 2004; Muise et al., 2007; Muise and Rotin, 2008). However, there is also evidence that implicates LAR-RPTPs and its synaptic interacting proteins in neurological disorders. Here we will discuss briefly the main human neurological conditions induced by LAR-RPTPs dysfunction and the related phenotypes observed in LARRPTPs knockout mice, which are summarized in Table $\mathbf{1 .}$

\section{PTPRF}

Decreased expression of PTPRF has been observed in induced pluripotent stem cells (iPSCs) obtained from Huntington's disease (HD) patients, which could have a role in some pathological features of this diseases, such as neural dysfunction and cell death (The HD iPSC Consortium, 2012). In this study, it was observed that HD iPSCs showed significantly less binding to the actin cytoskeleton compared to control iPSCs, in addition to showing cell-cell adhesion deficits in culture. The loss of PTPRF phosphatase activity has previously been associated with a decrease in focal adhesions (Sarhan et al., 2016a), which could affect the interaction of the actin cytoskeleton with the extracellular proteins (Wehrle-Haller, 2012). Therefore, the decrease in actin stability and increased actin dynamics in HD iPSC could be the result of reduced number of focal adhesions. Although it is not known if the reduced expression of PTPRF in HD could have a causative role for this disorder, it might be contributing to the progression of the disease by impairing the ability of neural cells to survive and differentiate in a correct way as a result of alterations in cell adhesion. In neuronal ceroid lipofuscinoses (NCL), a neurodegenerative disorder characterized by blindness, dementia and cortical atrophy, the absence of the soluble lysosomal palmitoylthioesterases $\mathrm{Cln} 1$ and $\mathrm{Cln} 5$, induces a significant reduction in the expression of PTRPF. Cortical transcription profiles of $\mathrm{Cln} 1$ and $\mathrm{Cln} 5$ deficient animals revealed profound alterations in genes related to protein phosphorylation that affect the dynamics of the cytoskeleton and neuronal growth cones. Intermediary proteins of these signaling pathways also showed an altered subcellular distribution in culture and brain tissue assays. Although the impairment of genes that control the balance of cytoskeletal dynamics such as PTPRF are not the only cause of NCL, they prove to be important components that contribute to the pathogenesis behind neurodegeneration (von Schantz et al.,
2008). Also, in immune-mediated demyelinating diseases, PTPRF expression is upregulated in exosomes obtained from patient cerebrospinal fluid, which is associated with the development of demyelinating diseases, and has been proposed as a biomarker for its early diagnosis (He et al., 2019).

PTPRF deficient mice have been developed to study PTPRF dysfunction. It has been observed that PTPRF knockout animals have a reduced number of cholinergic neurons in the forebrain and a reduced innervation of this cells to the hippocampus, an effect that was also observed in mice lacking PTPRF intracellular domain (Yeo et al., 1997; Van Lieshout et al., 2001). PTPRF deficiency also leads to a behavioral phenotype including induced spatial learning impairments and hyperactivity (Kolkman et al., 2004). Bernabeu and colleagues (2006) also showed that mice lacking PTPRF expression have an increased neurogenesis and increased NPC proliferation in the adult hippocampus (Bernabeu et al., 2006), suggesting a differential role for PTPRF in the forebrain and the hippocampus. Also, PTPRF knockout mice show reduced regenerative and collateral axonal sprouting in peripheral nerves and the forebrain (Van der Zee et al., 2003), and developmental impairments in the mammary gland cells, urogenital malformations, and an impaired craniofacial morphogenesis (Schaapveld et al., 1997; Uetani et al., 2009; Stewart et al., 2013). These animal model studies suggest an important role for PTPRF in the basal forebrain cholinergic signaling, hippocampal neurogenesis, axonal regeneration, and stem cell differentiation.

\section{PTPRD}

PTPRD mutations have been directly related to neurological disorders such as restless legs syndrome (RLS) (Schormair et al., 2008; Winkelmann et al., 2011; Yang et al., 2011), obsessive-compulsive disorder (OCD) (Mattheisen et al., 2015; Gazzellone et al., 2016), autism spectrum disorders (ASDs) (Pinto et al., 2010; Levy et al., 2011; Gai et al., 2012; Liu et al., 2016; Ji et al., 2021), attention deficit and hyperactivity disorder (ADHD) (Elia et al., 2010), schizophrenia (Li et al., 2018), intellectual disabilities (Choucair et al., 2015), bipolar disorder (Malhotra et al., 2011), addictions (Drgon et al., 2010; Johnson et al., 2008; Uhl et al., 2008a; Uhl et al., 2008b; Uhl et al., 2010), and tauopathies such as Alzheimer's disease (AD) (Chibnik et al., 2018). The main PTPRD genetic alterations observed in patients have been summarized in Table 2 .

One of the brain disorders in which PTPRD mutations have an important functional impact is RLS, also known as Willis-Ekbom disorder, which is characterized by symptoms including an urge to move, usually accompanied by uncomfortable sensations in the lower limbs (Schormair et al., 2008). The etiology has not yet been determined but may be related to dopaminergic and iron imbalance (Allen, 2004). The relationship between PTPRD and RLS has been established by genome wide association studies (GWAS), where single nucleotide polymorphisms (SNPs) frequently lead to reduced PTPRD mRNA expression (Drgonova and Walther, 2015; Hawrylycz et al., 2012). It is important to note that RLS is effectively treated with dopamine agonists and GABA analogs (Nagandla and De, 
TABLE 2 | Brain disorders induced by PTPRD mutations. Mutations in PTPRD have been associated with the development of several brain disorders such as intellectual disabilities, ASD, ADHD, OCD, schizophrenia, RLS, AD, and drug addictions. PTPRD genetic variations and its genomic location observed for each brain disorder are summarized.

\begin{tabular}{|c|c|c|c|}
\hline Disorder & Genetic variation & Location & References \\
\hline $\begin{array}{l}\text { Intellectual } \\
\text { Disability }\end{array}$ & CNV - Homozygous Deletion & $9 p 22.3$ & Choucair et al. (2015) \\
\hline ASD & CNV - Hemizygous Deletion & $\mathrm{n} / \mathrm{a}$ & Gai et al. (2012), Liu et al. (2016) \\
\hline ADHD & CNV - Hemizygous Deletion & $\begin{array}{l}\text { Start }=9,084,805, \text { end }=9,178,865 \\
\text { Start }=9,168,137, \text { end }=10,067,180 \\
\text { Start }=9,985,938, \text { end }=10,020,458\end{array}$ & Elia et al. (2010) \\
\hline OCD & $\begin{array}{l}\text { CNV - Duplication } \\
\text { SNP }\end{array}$ & $\begin{array}{c}9 \mathrm{p} 24.1 \\
1.28 \mathrm{Mb} \text { from the } 5^{\prime} \text { end of PTPRD }\end{array}$ & $\begin{array}{l}\text { Gazzellone et al. (2016) } \\
\text { Mattheisen et al. (2015) }\end{array}$ \\
\hline Schizophrenia & SNP & $\mathrm{n} / \mathrm{a}$ & Li et al. (2018) \\
\hline RLS & SNP & 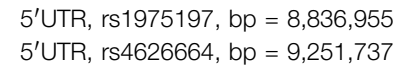 & Schormair et al. (2008) \\
\hline$A D$ & SNP & rs560380, bp = 9,112,698 & Chibnik et al. (2018) \\
\hline Drug Addiction & SNP & $\begin{array}{c}\text { rs12001948 } \\
\text { rs7854145 } \\
\text { rs2221184 } \\
\text { rs10511496 }\end{array}$ & Uhl et al. (2008a), Uhl, et al. (2008b) \\
\hline
\end{tabular}

2013), although whether PTPRD mutations impair dopaminergic neurotransmission remains unclear.

In OCD, a psychiatric disorder characterized by compulsive behaviors that patients perform in response to obsessive thoughts, $P T P R D$ variants have been associated with this disorder in two GWAS studies. These include a SNP $1.28 \mathrm{Mb}$ from $5^{\prime}$ end of PTPRD gene (Mattheisen et al., 2015), and copy number variants (CNVs) arising from a duplication of $1.5 \mathrm{Mb}$ at $9 \mathrm{p} 24.1$ (Gazzellone et al., 2016). Although there is no information on how these variants affect the expression of PTPRD, mouse models deficient for PTPRD show impairments in learning and memory tasks (Uetani et al., 2000), which is relevant for OCD, since memory impairments have been previously reported in OCD patients (Jaafari et al., 2013). Interestingly, PTPRD duplications may also contribute to the development of neurological disorders. It has been observed that duplication (71391bp) of the PTPRD gene at 9p23 has been related to an increased risk of suffering bipolar disorder (Malhotra et al., 2011), suggesting that increased PTPRD expression could also be involved in brain pathologies.

In brain disorders such as ASD, ADHD and intellectual disability, CNVs or mutations in the PTPRD gene (Elia et al., 2010; Pinto et al., 2010; Levy et al., 2011; Choucair et al., 2015) are directly associated with neurodevelopmental impairments. This is consistent with the phenotype of PTPRD knockout mice, which show impaired neuronal differentiation and disrupted cortical organization (Tomita et al., 2020). These mice also display reduced IL1RAPL1-mediated synapse formation (Yoshida et al., 2011), presumably because PTPRD knockout induces a significant reduction in IL1RAPL1 expression (Choucair et al., 2015; Park et al., 2020). Furthermore, specific mutation of the PTPRD IL1RAPL1-interacting domain induces hyperactivity and sleep disturbance, which is also observed in PTPRD conditional knockout mice (Park et al., 2020). Finally, PTPRD knockout mice show body growth retardation and spatial learning and memory impairments, which surprisingly correlates with an enhanced synaptic transmission in the hippocampus (Uetani et al., 2000).
In $\mathrm{AD}$, an $\mathrm{SNP}$ in the PTPRD locus shows a mild association with disease, but significant association with accumulation of neurofibrillary tangles (Chibnik et al., 2018). This may be induced by increased levels of phosphorylated tau protein in a PTPRD dependent mechanism, since its participation in tau phosphorylation signaling pathway has been previously suggested (Mitchell et al., 2016), and impaired PTPRD catalytic activity or expression could reduce tau dephosphorylation, although this hypothesis has not yet been tested.

\section{PTPRS}

Although no brain pathologies have been causally linked to PTPRS mutations or impaired function, in a rat model of Amyotrophic lateral sclerosis (ALS), reduced neuronal expression of PTPRS has been observed. This may inhibit axonal regeneration and favor glial scar formation through its interaction with CSPGs (Ohtake and Li, 2015; Shijo et al., 2018). Also, PTPRS has recently been considered as a potential target for AD treatment ( $\mathrm{Gu}$ et al., 2016). PTPRS interacts with amyloid precursor protein (APP) in the brain, and by knocking PTPRS expression, the affinity between $\beta$-secretase and APP can be reduced, decreasing $A \beta$ extracellular accumulation and inhibiting tau aggregation without affecting $\beta$-secretase enzymatic activity. Moreover, PTPRS knockout rescues behavioral impairments observed in an AD mice model ( $\mathrm{Gu}$ et al., 2016). This suggests PTPRS as a potential target for selective pharmacological intervention in AD.

As mentioned earlier, mice lacking PTPRS expression show body growth retardation, an abnormal physiology of the posterior pituitary, reduced hormone-releasing cells in the hypothalamus and reduced cholinergic neurons in the forebrain, which associates with neurological impairments such as spastic movements, tremor, ataxic gait, abnormal limb flexion and defective proprioception (Elchebly et al., 1999; Wallace et al., 1999). Moreover, PTPRS knockout mouse show an atypical hippocampus morphology, and a reduced thickness in the 
corpus callosum and brain cortex, which is suggested to be a consequence of a delayed neurodevelopment and an abnormal NPCs biology (Meathrel et al., 2002; Kirkham et al., 2006). On the other hand, Horn and colleagues (2012) have observed that PTRPS knockout mice show increased dendritic spine density and length, increased frequency of mEPSC, a greater paired-pulse facilitation, and a reduced long-term potentiation in the hippocampus, which correlates with an increased recognition memory, assessed by the novel object recognition test (Horn et al., 2012).

\section{Other Implications}

The spectrum of brain disorders involving LAR-RPTPs becomes wider when we consider that their functions in neural cells depend on their interactions. LAR-RPTP synaptic partners such as Slitrks, TrkC and IL1RAPL1, have also been implicated in neurological disorders (Um and Ko, 2013). For instance, Slitrk mutations have been associated with Tourette's syndrome, trichotillomania, schizophrenia, ASD and epilepsy (Piton et al., 2011; Wu et al., 2013). TrkC mutations have been found in patients with $\mathrm{AD}$, depression, bipolar disorder, and ADHD (Otnæss et al., 2009; Amador-Arjona et al., 2010). IL1RAPL1 has been related to a form of X-linked intellectual disability (Behnecke et al., 2011; Youngs et al., 2012). Hence, the links between LAR-RPTP impairments and the development of neurological disorders, combined with the evidence associating impairments in the LAR-RPTPs synaptic partners with neuronal pathologies, open a wide spectrum of brain disorders where LARRPTPs may be either directly or indirectly related. Finally, it is important to highlight that, impairments in the regulation of LAR-RPTP alternative splicing also induce psychiatric disorders. Neuronal micro-exons in LAR-RPTPs are dysregulated in brain samples from patients with ASD, suggesting that disruption of a coordinated program of LAR-RPTP splicing events may lead to neurodevelopmental pathologies (Irimia et al., 2014; QuesnelVallières et al., 2015). Therefore, the manipulation of alternative splicing machinery could be used as a therapeutical approach to restore the impaired neural development and the inhibitory/ excitatory imbalance observed in neurodevelopmental pathologies such as ASD (Nelson and Valakh, 2015; Parenti et al., 2020; Yoshida et al., 2021).

In summary, LAR-RPTPs are implicated in a variety of neurodevelopmental and neurological dysfunctions, and their mutations and impaired expression is associated with cognitive impairment to dementia. Further studies are necessary to understand how LAR-RPTP genetic variants can contribute to generate diverse brain pathologies. Considering that LAR-RPTPs encode extracellular interaction and intracellular catalytic and regulatory domains, variants in these regions may have diverse impact on neurobiology. Moreover, LAR-RPTPs alternative splicing variants are dependent on extremely short sequences (micro-exons), and SNPs in these critical regions could have profound consequences on LAR-RPTP neurological functions. For example, in the case of LAR-RPTP contributions to synaptic differentiation and function, even subtle genetic alterations could impair interactions with synaptic interaction partners, directly affecting the number of excitatory or inhibitory synapses. Thus,

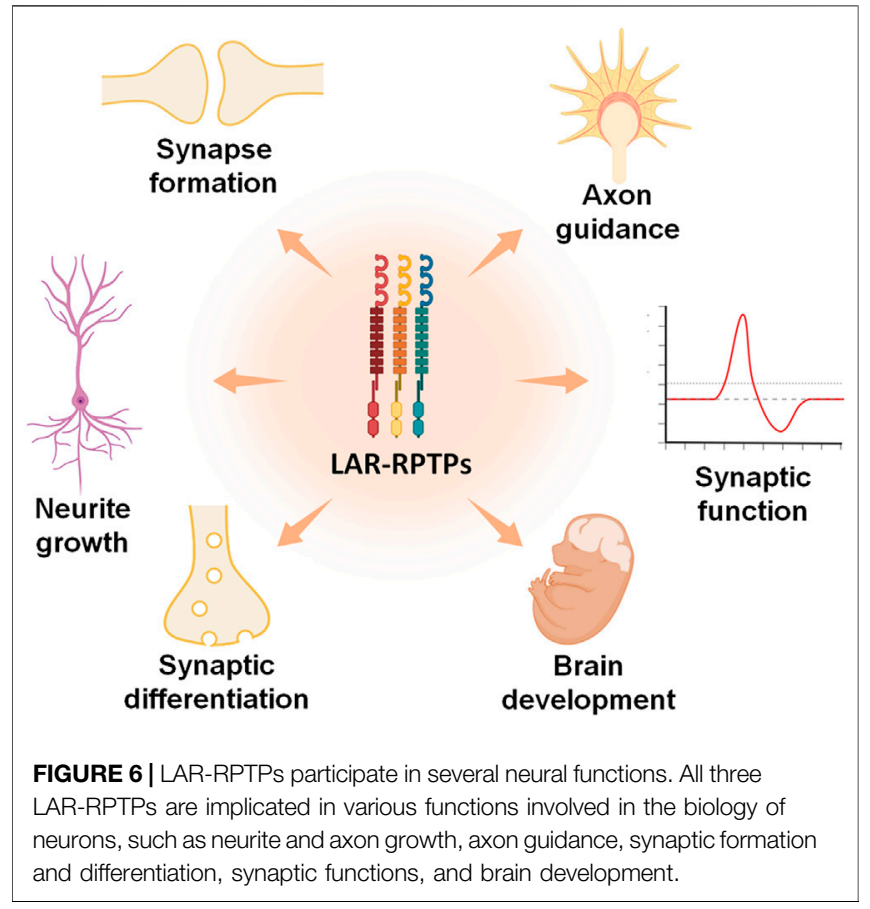

LAR-RPTP genetic variants may contribute in myriad ways to neurological processes that are disrupted in various neuropsychiatric disorders.

\section{CONCLUDING REMARKS}

All the evidence summarized here confirms an important neurological role for LAR-RPTPs in health and disease. Although LAR-RPTP functions as cell adhesion molecules are well established in neurobiology, there are several lines of evidence that describes their phosphatase activity as an important modulator of neurite growth, axon guidance, synapse formation and differentiation, synaptic function, and brain development (Figure 6).

However, it is important to note that the functions of the different LAR-RPTPs in the brain are not yet fully understood, which has been illustrated by the latest research demonstrating phenotypes different from those previously observed (Han et al., 2020c; Sclip and Südhof, 2020). Given the many processes in which LAR-RPTPs participate, their expression and function must be tightly regulated. Therefore, it is important to consider all the variables that could alter LAR-RPTPs when studying them. Some of these variables are the stage of development and the cell type; in the developing mouse brain, PTPRF expression is dramatically reduced as neuroblasts differentiate and migrate (Schaapveld et al., 1998). On the other hand, PTPRD and PTPRS are highly expressed as neural cells differentiate (Schaapveld et al., 1998), but PTPRS expression is higher in the embryonic nervous system, with a parallel expression pattern to PTPRD 
(Chagnon et al., 2004). Also, the PTPRD isoform switch from including meA3 to including meA9 between E18.5 and P4 (Wamsley et al., 2018).

Moreover, PTPRD meB has been reported to be highly included at embryonic brain samples (E11.5), suggesting that the PTPRD trans-synaptic interactions promoted by $\mathrm{meB}$ inclusion may have a role in synaptogenesis during brain development (Yoshida et al., 2011; Yamagata et al., 2015). On the other hand, it has been recently reported that three PTPRD micro-exons that code for meA and meB are differentially included between inhibitory and excitatory neurons, suggesting that the alternative splicing of meA/B is finely tuned across neuronal cell types (Parada et al., 2021). Thus, LAR-RPTPs differential expression pattern suggests that they could exert different roles in the developing brain cells and in their function. By considering LAR-RPTPs regional and temporal expression patterns, we could study their physiological phenotype more specifically.

The evidence summarized here evidence that LAR-RPTPs are not only important molecules for shaping the synapse, but also for brain development and for its physiological functions. As a result, alterations in LAR-RPTP expression and function are associated with different brain disorders. Indeed, the diverse neurological processes in which LAR-RPTPs participate highlights the importance of studying the role of specific LARRPTP functions such as ligand binding and/or phosphatase activity, and how these are disrupted in brain disorders. Thus, elaborating approaches to modulate specific LAR-RPTP

\section{REFERENCES}

Aicher, B., Lerch, M. M., Müller, T., Schilling, J., and Ullrich, A. (1997). Cellular Redistribution of Protein Tyrosine Phosphatases LAR and PTP $\sigma$ by Inducible Proteolytic Processing. J. Cell Biol. 138, 681-696. doi:10.1083/jcb.138.3.681

Allen, R. (2004). Dopamine and Iron in the Pathophysiology of Restless Legs Syndrome (RLS). Sleep Med. 5, 385-391. doi:10.1016/j.sleep.2004.01.012

Amador-Arjona, A., Delgado-Morales, R., Belda, X., Gagliano, H., Gallego, X., Keck, M. E., et al. (2010). Susceptibility to Stress in Transgenic Mice Overexpressing TrkC, a Model of Panic Disorder. J. Psychiatr. Res. 44, 157-167. doi:10.1016/J.JPSYCHIRES.2009.07.008

Ball, R. W., Warren-Paquin, M., Tsurudome, K., Liao, E. H., Elazzouzi, F., Cavanagh, C., et al. (2010). Retrograde BMP Signaling Controls Synaptic Growth at the NMJ by Regulating Trio Expression in Motor Neurons. Neuron 66, 536-549. doi:10.1016/J.NEURON.2010.04.011

Bamji, S. X., Rico, B., Kimes, N., and Reichardt, L. F. (2006). BDNF Mobilizes Synaptic Vesicles and Enhances Synapse Formation by Disrupting Cadherin$\beta$-Catenin Interactions. J. Cell Biol. 174, 289-299. doi:10.1083/jcb.200601087

Behnecke, A., Hinderhofer, K., Bartsch, O., Nümann, A., Ipach, M.-L., Damatova, N., et al. (2011). Intragenic Deletions of IL1RAPL1: Report of Two Cases and Review of the Literature. Am. J. Med. Genet. 155, 372-379. doi:10.1002/ ajmg.a.33656

Bernabeu, R., Yang, T., Xie, Y., Mehta, B., Ma, S. Y., and Longo, F. M. (2006). Downregulation of the LAR Protein Tyrosine Phosphatase Receptor Is Associated with Increased Dentate Gyrus Neurogenesis and an Increased Number of Granule Cell Layer Neurons. Mol. Cell Neurosci. 31, 723-738. doi:10.1016/j.mcn.2006.01.003

Bomkamp, C., Padmanabhan, N., Karimi, B., Ge, Y., Chao, J. T., Loewen, C. J. R., et al. (2019). Mechanisms of PTPб-Mediated Presynaptic Differentiation. Front. Synaptic Neurosci. 11, 1-21. doi:10.3389/fnsyn.2019.00017 functions could be an important therapeutic strategy for several brain disorders.

\section{AUTHOR CONTRIBUTIONS}

FC and BIC wrote the manuscript. GMF and GIC conceived and supervised the project. All authors contributed to the article and approved the submitted version.

\section{FUNDING}

This work was funded by ANID FONDECYT regular 1161374 and 1210507 grants, an ANID/PCI REDES180113 grant, a Universidad Mayor Startup project, and an IBRO return home fellowship to GIC. FC was funded by Universidad Mayor postdoctoral fellowship and ANID FONDECYT postdoctorado 3190517. BC was funded by Universidad Mayor PhD fellowship. GMF was supported by a Wellcome Trust/Royal Society Sir Henry Dale Fellowship 211209/Z/18/Z.

\section{ACKNOWLEDGMENTS}

We would like to acknowledge the excellent advice of Dr. Guillermo Parada regarding alternative splicing mechanisms, which contributed to the development of this review.

Brigidi, G. S., and Bamji, S. X. (2011). Cadherin-catenin Adhesion Complexes at the Synapse. Curr. Opin. Neurobiol. 21, 208-214. doi:10.1016/ J.CONB.2010.12.004

Chagnon, M. J., Uetani, N., and Tremblay, M. L. (2004). Functional Significance of the LAR Receptor Protein Tyrosine Phosphatase Family in Development and Diseases. Biochem. Cell Biol. 82, 664-675. doi:10.1139/ o04-120

Chagnon, M. J., Wu, C.-L., Nakazawa, T., Yamamoto, T., Noda, M., Blanchetot, C., et al. (2010). Receptor Tyrosine Phosphatase Sigma (RPTPo) Regulates, p250GAP, a Novel Substrate that Attenuates Rac Signaling. Cell Signal. 22, 1626-1633. doi:10.1016/J.CELLSIG.2010.06.001

Chen, M. J., Dixon, J. E., and Manning, G. (2017). Genomics and Evolution of Protein Phosphatases. Sci. Signal. 10, 1-18. doi:10.1126/ scisignal.aag 1796

Chibnik, L. B., White, C. C., Mukherjee, S., Raj, T., Yu, L., Larson, E. B., et al. (2018). Susceptibility to Neurofibrillary Tangles: Role of the PTPRD Locus and Limited Pleiotropy with Other Neuropathologies. Mol. Psychiatry 23, 1521-1529. doi:10.1038/mp.2017.20

Chien, P. N., and Ryu, S. E. (2013). Protein Tyrosine Phosphatase $\sigma$ in Proteoglycan-Mediated Neural Regeneration Regulation. Mol. Neurobiol. 47, 220-227. doi:10.1007/s12035-012-8346-X

Choi, Y., Nam, J., Whitcomb, D. J., Song, Y. S., Kim, D., Jeon, S., et al. (2016). SALM5 Trans-synaptically Interacts with LAR-RPTPs in a Splicing-dependent Manner to Regulate Synapse Development. Sci. Rep. 6, 26676. doi:10.1038/ srep26676

Choucair, N., Mignon-Ravix, C., Cacciagli, P., Abou Ghoch, J., Fawaz, A., Mégarbané, A., et al. (2015). Evidence that Homozygous PTPRD Gene Microdeletion Causes Trigonocephaly, Hearing Loss, and Intellectual Disability. Mol. Cytogenet. 8, 1-8. doi:10.1186/s13039-015-0149-0

Cohen, P. (2002). The Origins of Protein Phosphorylation. Nat. Cell Biol. 4, E127-E130. doi:10.1038/ncb0502-e127 
Coles, C. H., Jones, E. Y., and Aricescu, A. R. (2015). Extracellular Regulation of Type IIa Receptor Protein Tyrosine Phosphatases: Mechanistic Insights from Structural Analyses. Semin. Cell Developmental Biol. 37, 98-107. doi:10.1016/ j.semcdb.2014.09.007

Coles, C. H., Shen, Y., Tenney, A. P., Siebold, C., Sutton, G. C., Lu, W., et al. (2011). Proteoglycan-Specific Molecular Switch for RPTPo Clustering and Neuronal Extension. Science 332, 484-488. doi:10.1126/science.1200840

Dai, J., Aoto, J., and Südhof, T. C. (2019). Alternative Splicing of Presynaptic Neurexins Differentially Controls Postsynaptic NMDA and AMPA Receptor Responses. Neuron 102, 993-1008. e5. doi:10.1016/j.neuron.2019.03.032

Debant, A., Serra-Pages, C., Seipel, K., O’Brien, S., Tang, M., Park, S. H., et al. (1996). The Multidomain Protein Trio Binds the LAR Transmembrane Tyrosine Phosphatase, Contains a Protein Kinase Domain, and Has Separate Rac-specific and Rho-specific Guanine Nucleotide Exchange Factor Domains. Proc. Natl. Acad. Sci. 93, 5466-5471. doi:10.1073/PNAS.93.11.5466

Dickinson, B. A., Jo, J., Seok, H., Son, G. H., Whitcomb, D. J., Davies, C. H., et al. (2009). A Novel Mechanism of Hippocampal LTD Involving Muscarinic Receptor-Triggered Interactions between AMPARs, GRIP and Liprin- $\alpha$. Mol. Brain 2, 18. doi:10.1186/1756-6606-2-18

Drgon, T., Zhang, P.-W., Johnson, C., Walther, D., Hess, J., Nino, M., et al. (2010). Genome Wide Association for Addiction: Replicated Results and Comparisons of Two Analytic Approaches. PLoS One 5, e8832. doi:10.1371/ journal.pone.0008832

Drgonova, J., Walther, D., Wang, K. J., Hartstein, G. L., Lochte, B., Troncoso, J., et al. (2015). Mouse Model for Protein Tyrosine Phosphatase D (PTPRD) Associations with Restless Leg Syndrome or Willis-Ekbom Disease and Addiction: Reduced Expression Alters Locomotion, Sleep Behaviors and Cocaine-Conditioned Place Preference. Mol. Med. 21, 717-725. doi:10.2119/ molmed.2015.00017

Dunah, A. W., Hueske, E., Wyszynski, M., Hoogenraad, C. C., Jaworski, J., Pak, D. T., et al. (2005). LAR Receptor Protein Tyrosine Phosphatases in the Development and Maintenance of Excitatory Synapses. Nat. Neurosci. 8, 458-467. doi:10.1038/nn1416

Dyck, S. M., Alizadeh, A., Santhosh, K. T., Proulx, E. H., Wu, C.-L., and KarimiAbdolrezaee, S. (2015). Chondroitin Sulfate Proteoglycans Negatively Modulate Spinal Cord Neural Precursor Cells by Signaling through LAR and RPTP $\sigma$ and Modulation of the Rho/ROCK Pathway. Stem Cells 33, 2550-2563. doi:10.1002/ stem.1979

Elchebly, M., Wagner, J., Kennedy, T. E., Lanctôt, C., Michaliszyn, E., Itié, A., et al. (1999). Neuroendocrine Dysplasia in Mice Lacking Protein Tyrosine Phosphatase $\sigma$. Nat. Genet. 21, 330-333. doi:10.1038/6859

E.L. Craig, S., and M. Brady-Kalnay, S. (2011). Tumor-derived Extracellular Fragments of Receptor Protein Tyrosine Phosphatases (RPTPs) as Cancer Molecular Diagnostic Tools. Acamc 11, 133-140. doi:10.2174/ 187152011794941244

Elia, J., Gai, X., Xie, H. M., Perin, J. C., Geiger, E., Glessner, J. T., et al. (2010). Rare Structural Variants Found in Attention-Deficit Hyperactivity Disorder Are Preferentially Associated with Neurodevelopmental Genes. Mol. Psychiatry 15, 637-646. doi:10.1038/mp.2009.57

Fisher, D., Xing, B., Dill, J., Li, H., Hoang, H. H., Zhao, Z., et al. (2011). Leukocyte Common Antigen-Related Phosphatase Is a Functional Receptor for Chondroitin Sulfate Proteoglycan Axon Growth Inhibitors. J. Neurosci. 31, 14051-14066. doi:10.1523/JNEUROSCI.1737-11.2011

Fry, E. J., Chagnon, M. J., López-Vales, R., Tremblay, M. L., and David, S. (2009). Corticospinal Tract Regeneration after Spinal Cord Injury in Receptor Protein Tyrosine Phosphatase Sigma Deficient Mice. Glia 58, NA. doi:10.1002/ glia. 20934

Fuentes-Medel, Y., and Budnik, V. (2010). Ménage à Trio during BMP-Mediated Retrograde Signaling at the NMJ. Neuron 66, 473-475. doi:10.1016/ J.NEURON.2010.05.016

Fukai, S., and Yoshida, T. (2020). Roles of Type IIa Receptor Protein Tyrosine Phosphatases as Synaptic Organizers. FEBS J. doi:10.1111/febs.15666in press

Gai, X., Xie, H. M., Perin, J. C., Takahashi, N., Murphy, K., Wenocur, A. S., et al. (2012). Rare Structural Variation of Synapse and Neurotransmission Genes in Autism. Mol. Psychiatry 17, 402-411. doi:10.1038/mp.2011.10

Gazzellone, M. J., Zarrei, M., Burton, C. L., Walker, S., Uddin, M., Shaheen, S. M., et al. (2016). Uncovering Obsessive-Compulsive Disorder Risk Genes in a
Pediatric Cohort by High-Resolution Analysis of Copy Number Variation. J. Neurodevelop Disord. 8, 36. doi:10.1186/s11689-016-9170-9

Goto-Ito, S., Yamagata, A., Sato, Y., Uemura, T., Shiroshima, T., Maeda, A., et al. (2018). Structural Basis of Trans-synaptic Interactions between PTP $\delta$ and SALMs for Inducing Synapse Formation. Nat. Commun. 9, 269. doi:10.1038/s41467-017-02417-z

Groen, A., Lemeer, S., Van Der Wijk, T., Overvoorde, J., Heck, A. J. R., Ostman, A., et al. (2005). Differential Oxidation of Protein-Tyrosine Phosphatases. J. Biol. Chem. 280, 10298-10304. doi:10.1074/jbc.M412424200

Groen, A., Overvoorde, J., van der Wijk, T., and den Hertog, J. (2008). Redox Regulation of Dimerization of the Receptor Protein-Tyrosine Phosphatases RPTPa, LAR, RPTP $\mu$ and CD45. FEBS J. 275, 2597-2604. doi:10.1111/j.17424658.2008.06407.x

Groß, S., Knebel, A., Tenev, T., Neininger, A., Gaestel, M., Herrlich, P., et al. (1999). Inactivation of Protein-Tyrosine Phosphatases as Mechanism of UV-Induced Signal Transduction. J. Biol. Chem. 274, 26378-26386. doi:10.1074/ jbc.274.37.26378

Gu, Y., Shu, Y., Corona, A., Xu, K., Yi, A. F., Chen, S., et al. (2016). Alzheimer's Disease Pathogenesis Is Dependent on Neuronal Receptor PTPsigma. bioRxiv [Preprint]. Available at: https://www.biorxiv.org/content/10.1101/079806v4 (Accessed October 16, 2020).

Haapasalo, A., Kim, D. Y., Carey, B. W., Turunen, M. K., Pettingell, W. H., and Kovacs, D. M. (2007). Presenilin $/ \gamma$-Secretase-mediated Cleavage Regulates Association of Leukocyte-Common Antigen-Related (LAR) Receptor Tyrosine Phosphatase with $\beta$-Catenin. J. Biol. Chem. 282, 9063-9072. doi:10.1074/jbc.M611324200

Hamasaki, H., Fujitani, M., and Yamashita, T. (2016). NME2 Associates with PTP $\sigma$ to Transduce Signals from Chondroitin Sulfate Proteoglycans. Biochem. Biophysical Res. Commun. 471, 522-527. doi:10.1016/j.bbrc.2016.02.042

Han, K. A., Jeon, S., Um, J. W., and Ko, J. (2016). Emergent Synapse Organizers: LAR-RPTPs and Their Companions. Int. Rev. Cell Mol. Biol. 324, 39-65. doi:10.1016/BS.IRCMB.2016.01.002

Han, K. A., Kim, Y.-J., Yoon, T. H., Kim, H., Bae, S., Um, J. W., et al. (2020a). LARRPTPs Directly Interact with Neurexins to Coordinate Bidirectional Assembly of Molecular Machineries. J. Neurosci. 40, 8438-8462. doi:10.1523/ JNEUROSCI.1091-20.2020

Han, K. A., Ko, J. S., Pramanik, G., Kim, J. Y., Tabuchi, K., Um, J. W., et al. (2018). PTPo Drives Excitatory Presynaptic Assembly via Various Extracellular and Intracellular Mechanisms. J. Neurosci. 38, 6700-6721. doi:10.1523/ JNEUROSCI.0672-18.2018

Han, K. A., Lee, H.-Y., Lim, D., Shin, J., Yoon, T. H., Lee, C., et al. (2020b). PTPб Controls Presynaptic Organization of Neurotransmitter Release Machinery at Excitatory Synapses. iScience 23, 101203. doi:10.1016/j.isci.2020.101203

Han, K. A., Lee, H.-Y., Lim, D., Shin, J., Yoon, T. H., Liu, X., et al. (2020c). Receptor Protein Tyrosine Phosphatase delta Is Not Essential for Synapse Maintenance or Transmission at Hippocampal Synapses. Mol. Brain 13, 1-10. doi:10.1186/ s13041-020-00629-x

Hata, Y., Butz, S., and Sudhof, T. (1996). CASK: A Novel dlg/PSD95 Homolog with an N-Terminal Calmodulin-dependent Protein Kinase Domain Identified by Interaction with Neurexins. J. Neurosci. 16, 2488-2494. doi:10.1523/ jneurosci.16-08-02488.1996

Hawrylycz, M. J., Lein, E. S., Guillozet-Bongaarts, A. L., Shen, E. H., Ng, L., Miller, J. A., et al. (2012). An Anatomically Comprehensive Atlas of the Adult Human Brain Transcriptome. Nature 489, 391-399. doi:10.1038/nature11405

He, J., Ren, M., Li, H., Yang, L., Wang, X., and Yang, Q. (2019). Exosomal Circular RNA as a Biomarker Platform for the Early Diagnosis of Immune-Mediated Demyelinating Disease. Front. Genet. 10, 860. doi:10.3389/fgene.2019.00860

Horn, K. E., Xu, B., Gobert, D., Hamam, B. N., Thompson, K. M., Wu, C.-L., et al. (2012). Receptor Protein Tyrosine Phosphatase Sigma Regulates Synapse Structure, Function and Plasticity. J. Neurochem. 122, 147-161. doi:10.1111/ j.1471-4159.2012.07762.x

Irimia, M., Weatheritt, R. J., Ellis, J. D., Parikshak, N. N., Gonatopoulos-Pournatzis, T., Babor, M., et al. (2014). A Highly Conserved Program of Neuronal Microexons Is Misregulated in Autistic Brains. Cell 159, 1511-1523. doi:10.1016/j.cell.2014.11.035

Jaafari, N., Frasca, M., Rigalleau, F., Rachid, F., Gil, R., Olié, J.-P., et al. (2013). for Insight Study Group, et alForgetting what you have checked: a link between 
working memory impairment and checking behaviors in obsessive-compulsive disorder. Eur. Psychiatr. 28 (2), 87-93. doi:10.1016/j.eurpsy.2011.07.001

Ji, G., Li, S., Ye, L., and Guan, J. (2021). Gene Module Analysis Reveals Cell-type Specificity and Potential Target Genes in Autism's Pathogenesis. Biomedicines 9, 410. doi:10.3390/biomedicines9040410

Johnson, C., Drgon, T., Liu, Q.-R., Zhang, P.-W., Walther, D., Li, C.-Y., et al. (2008). Genome Wide Association for Substance Dependence: Convergent Results from Epidemiologic and Research Volunteer Samples. BMC Med. Genet. 9, 113. doi:10.1186/1471-2350-9-113

Johnson, K. G., Tenney, A. P., Ghose, A., Duckworth, A. M., Higashi, M. E., Parfitt, K., et al. (2006). The HSPGs Syndecan and Dallylike Bind the Receptor Phosphatase LAR and Exert Distinct Effects on Synaptic Development. Neuron 49, 517-531. doi:10.1016/J.NEURON.2006.01.026

Kaufmann, N., DeProto, J., Ranjan, R., Wan, H., and Van Vactor, D. (2002). Drosophila Liprin- $\alpha$ and the Receptor Phosphatase Dlar Control Synapse Morphogenesis. Neuron 34, 27-38. doi:10.1016/S0896-6273(02)00643-8

Kim, K., Shin, W., Kang, M., Lee, S., Kim, D., Kang, R., et al. (2020). Presynaptic PTPo Regulates Postsynaptic NMDA Receptor Function through Direct Adhesion-independent Mechanisms. Elife 9, 1-26. doi:10.7554/eLife.54224

Kirkham, D. L., Pacey, L. K., Axford, M. M., Siu, R., Rotin, D., and Doering, L. C. (2006). Neural Stem Cells from Protein Tyrosine Phosphatase Sigma Knockout Mice Generate an Altered Neuronal Phenotype in Culture. BMC Neurosci. 7, 50. doi:10.1186/1471-2202-7-50

Ko, J., Kim, S., Chung, H. S., Kim, K., Han, K., Kim, H., et al. (2006). SALM Synaptic Cell Adhesion-like Molecules Regulate the Differentiation of Excitatory Synapses. Neuron 50, 233-245. doi:10.1016/j.neuron.2006.04.005

Ko, J., Kim, S., Valtschanoff, J. G., Shin, H., Lee, J.-R., Sheng, M., et al. (2003a). Interaction between Liprin- $\alpha$ and GIT1 Is Required for AMPA Receptor Targeting. J. Neurosci. 23, 1667-1677. doi:10.1523/JNEUROSCI.23-0501667.2003

Ko, J., Na, M., Kim, S., Lee, J.-R., and Kim, E. (2003b). Interaction of the ERC Family of RIM-Binding Proteins with the Liprin- $\alpha$ Family of Multidomain Proteins. J. Biol. Chem. 278, 42377-42385. doi:10.1074/jbc.M307561200

Ko, J. S., Pramanik, G., Um, J. W., Shim, J. S., Lee, D., Kim, K. H., et al. (2015). PTP $\sigma$ Functions as a Presynaptic Receptor for the glypican-4/LRRTM4 Complex and Is Essential for Excitatory Synaptic Transmission. Proc. Natl. Acad. Sci. USA 112, 1874-1879. doi:10.1073/pnas.1410138112

Kolkman, M. J. M., Streijger, F., Linkels, M., Bloemen, M., Heeren, D. J., Hendriks, W. J. A. J., et al. (2004). Mice Lacking Leukocyte Common Antigen-Related (LAR) Protein Tyrosine Phosphatase Domains Demonstrate Spatial Learning Impairment in the Two-Trial Water Maze and Hyperactivity in Multiple Behavioural Tests. Behav. Brain Res. 154, 171-182. doi:10.1016/ J.BBR.2004.02.006

Kurihara, D., and Yamashita, T. (2012). Chondroitin Sulfate Proteoglycans DownRegulate Spine Formation in Cortical Neurons by Targeting TropomyosinRelated Kinase B (TrkB) Protein. J. Biol. Chem. 287, 13822-13828. doi:10.1074/ jbc.M111.314070

Kurihara, D., Yamashita, T., and Saito, H. (1990). Structural Diversity and Evolution of Human Receptor-like Protein Tyrosine Phosphatases. J. Biol. Chem. 10, 3241-3252.

Kwon, S.-K., Woo, J., Kim, S.-Y., Kim, H., and Kim, E. (2010). Trans-synaptic Adhesions between Netrin-G Ligand-3 (NGL-3) and Receptor Tyrosine Phosphatases LAR, Protein-Tyrosine Phosphatase $\delta$ (PTP $\delta$ ), and PTP $\sigma$ via Specific Domains Regulate Excitatory Synapse Formation. J. Biol. Chem. 285, 13966-13978. doi:10.1074/jbc.M109.061127

Kypta, R. M., Su, H., and Reichardt, L. F. (1996). Association between a Transmembrane Protein Tyrosine Phosphatase and the Cadherin-Catenin Complex. J. Cell Biol. 134, 1519-1529. doi:10.1083/jcb.134.6.1519

Lang, B. T., Cregg, J. M., DePaul, M. A., Tran, A. P., Xu, K., Dyck, S. M., et al. (2015). Modulation of the Proteoglycan Receptor PTPo Promotes Recovery after Spinal Cord Injury. Nature 518, 404-408. doi:10.1038/ nature 13974

Lee, H., Lee, E.-J., Song, Y. S., and Kim, E. (2014). Long-term Depression-Inducing Stimuli Promote Cleavage of the Synaptic Adhesion Molecule NGL-3 through NMDA Receptors, Matrix Metalloproteinases and Presenilin $/ \gamma$-Secretase. Phil. Trans. R. Soc. B 369, 20130158. doi:10.1098/rstb.2013.0158

Lesnikova, A., Casarotto, P. C., Fred, S. M., Voipio, M., Winkel, F., Steinzeig, A., et al. (2020). Chondroitinase and Antidepressants Promote Plasticity by
Releasing TRKB from Dephosphorylating Control of PTP $\sigma$ in Parvalbumin Neurons. J. Neurosci. 41, 972-980. doi:10.1523/JNEUROSCI.2228-20.2020

Levy, D., Ronemus, M., Yamrom, B., Lee, Y.-h., Leotta, A., Kendall, J., et al. (2011). Rare De Novo and Transmitted Copy-Number Variation in Autistic Spectrum Disorders. Neuron 70, 886-897. doi:10.1016/J.NEURON.2011.05.015

Li, J., Yoshikawa, A., Brennan, M. D., Ramsey, T. L., and Meltzer, H. Y. (2018). Genetic Predictors of Antipsychotic Response to Lurasidone Identified in a Genome Wide Association Study and by Schizophrenia Risk Genes. Schizophrenia Res. 192, 194-204. doi:10.1016/J.SCHRES.2017.04.009

Li, Y., Zhang, P., Choi, T.-Y., Park, S. K., Park, H., Lee, E.-J., et al. (2015). SplicingDependent Trans-synaptic SALM3-LAR-RPTP Interactions Regulate Excitatory Synapse Development and Locomotion. Cell Rep. 12, 1618-1630. doi:10.1016/j.celrep.2015.08.002

Liberti, S., Sacco, F., Calderone, A., Perfetto, L., Iannuccelli, M., Panni, S., et al. (2013). HuPho: the Human Phosphatase portal. FEBS J. 280, 379-387. doi:10.1111/j.1742-4658.2012.08712.x

Lie, E., Ko, J. S., Choi, S.-Y., Roh, J. D., Cho, Y. S., Noh, R., et al. (2016). SALM4 Suppresses Excitatory Synapse Development by Cis-Inhibiting Transsynaptic SALM3-LAR Adhesion. Nat. Commun. 7, 12328. doi:10.1038/ ncomms 12328

Lin, Z., Liu, J., Ding, H., Xu, F., and Liu, H. (2018). Structural Basis of SALM5Induced PTP $\delta$ Dimerization for Synaptic Differentiation. Nat. Commun. 9, 268. doi:10.1038/s41467-017-02414-2

Liu, X., Shimada, T., Otowa, T., Wu, Y.-Y., Kawamura, Y., Tochigi, M., et al. (2016). Genome-wide Association Study of Autism Spectrum Disorder in the East Asian Populations. Autism Res. 9, 340-349. doi:10.1002/aur.1536

Luo, L., Ambrozkiewicz, M. C., Benseler, F., Chen, C., Dumontier, E., Falkner, S., et al. (2020). Optimizing Nervous System-specific Gene Targeting with Cre Driver Lines: Prevalence of Germline Recombination and Influencing Factors. Neuron 106, 37-65. e5. doi:10.1016/j.neuron.2020.01.008

Mah, W., Ko, J., Nam, J., Han, K., Chung, W. S., and Kim, E. (2010). Selected SALM (Synaptic Adhesion-like Molecule) Family Proteins Regulate Synapse Formation. J. Neurosci. 30, 5559-5568. doi:10.1523/JNEUROSCI.4839-09.2010

Malhotra, D., McCarthy, S., Michaelson, J. J., Vacic, V., Burdick, K. E., Yoon, S., et al. (2011). High Frequencies of De Novo CNVs in Bipolar Disorder and Schizophrenia. Neuron 72, 951-963. doi:10.1016/J.NEURON.2011.11.007

Mattheisen, M., Samuels, J. F., Wang, Y., Greenberg, B. D., Fyer, A. J., McCracken, J. T., et al. (2015). Genome-wide Association Study in Obsessive-Compulsive Disorder: Results from the OCGAS. Mol. Psychiatry 20, 337-344. doi:10.1038/ mp.2014.43

McLean, J., Batt, J., Doering, L. C., Rotin, D., and Bain, J. R. (2002). Enhanced Rate of Nerve Regeneration and Directional Errors after Sciatic Nerve Injury in Receptor Protein Tyrosine Phosphatase $\varsigma$ Knock-Out Mice. J. Neurosci. 22, 5481-5491. doi:10.1523/jneurosci.22-13-05481.2002

Meathrel, K., Adamek, T., Batt, J., Rotin, D., and Doering, L. C. (2002). Protein Tyrosine Phosphatase ?-deficient Mice Show Aberrant Cytoarchitecture and Structural Abnormalities in the central Nervous System. J. Neurosci. Res. 70, 24-35. doi:10.1002/jnr.10382

Mitchell, C. J., Kim, M.-S., Zhong, J., Nirujogi, R. S., Bose, A. K., and Pandey, A. (2016). Unbiased Identification of Substrates of Protein Tyrosine Phosphatase Ptp-3 in C. elegans. Mol. Oncol. 10, 910-920. doi:10.1016/j.molonc.2016.03.003

Moon, S., and Zheng, Y. (2003). Rho GTPase-Activating Proteins in Cell Regulation. Trends Cell Biol. 13, 13-22. doi:10.1016/S0962-8924(02)00004-1

Morrison, C. D., and Münzberg, H. (2012). Capricious Cre: The Devil Is in the Details. Endocrinology 153, 1005-1007. doi:10.1210/en.2011-2169

Muise, A. M., Walters, T., Wine, E., Griffiths, A. M., Turner, D., Duerr, R. H., et al. (2007). Protein-Tyrosine Phosphatase Sigma Is Associated with Ulcerative Colitis. Curr. Biol. 17, 1212-1218. doi:10.1016/J.CUB.2007.06.013

Muise, A., and Rotin, D. (2008). Apical junction Complex Proteins and Ulcerative Colitis: a Focus on thePTPRSgene. Expert Rev. Mol. Diagn. 8, 465-477. doi:10.1586/14737159.8.4.465

Nagandla, K., and De, S. (2013). Restless Legs Syndrome: Pathophysiology and Modern Management. Postgrad. Med. J. 89, 402-410. doi:10.1136/ postgradmedj-2012-131634

Nakamura, F., Okada, T., Shishikura, M., Uetani, N., Taniguchi, M., Yagi, T., et al. (2017). Protein Tyrosine Phosphatase $\delta$ Mediates the Sema3A-Induced Cortical Basal Dendritic Arborization through the Activation of Fyn Tyrosine Kinase. J. Neurosci. 37, 7125-7139. doi:10.1523/JNEUROSCI.2519-16.2017 
Nakazawa, T., Watabe, A. M., Tezuka, T., Yoshida, Y., Yokoyama, K., Umemori, H., et al. (2003). p250GAP, a Novel Brain-Enriched GTPase-Activating Protein for Rho Family GTPases, Is Involved in theN-Methyl-D-Aspartate Receptor Signaling. MBoC 14, 2921-2934. doi:10.1091/mbc.E02-09-0623

Nam, H.-J., Poy, F., Krueger, N. X., Saito, H., and Frederick, C. A. (1999). Crystal Structure of the Tandem Phosphatase Domains of RPTP LAR. Cell 97, 449-457. doi:10.1016/S0092-8674(00)80755-2

Nelson, S. B., and Valakh, V. (2015). Excitatory/Inhibitory Balance and Circuit Homeostasis in Autism Spectrum Disorders. Neuron 87, 684-698. doi:10.1016/ j.neuron.2015.07.033

Ohtake, Y., and Li, S. (2015). Molecular Mechanisms of Scar-Sourced Axon Growth Inhibitors. Brain Res. 1619, 22-35. doi:10.1016/ J.BRAINRES.2014.08.064

Okabe, T., Nakamura, T., Nishimura, Y. N., Kohu, K., Ohwada, S., Morishita, Y., et al. (2003). RICS, a Novel GTPase-Activating Protein for Cdc42 and Rac1, Is Involved in the $\beta$-Catenin- $\mathrm{N}$-cadherin andN-Methyl-D-Aspartate Receptor Signaling. J. Biol. Chem. 278, 9920-9927. doi:10.1074/ jbc.M208872200

Olsen, O., Moore, K. A., Fukata, M., Kazuta, T., Trinidad, J. C., Kauer, F. W., et al. (2005). Neurotransmitter Release Regulated by a MALS-Liprin- $\alpha$ Presynaptic Complex. J. Cell Biol. 170, 1127-1134. doi:10.1083/jcb.200503011

Otnæss, M. K., Djurovic, S., Rimol, L. M., Kulle, B., Kähler, A. K., Jönsson, E. G., et al. (2009). Evidence for a Possible Association of Neurotrophin Receptor (NTRK-3) Gene Polymorphisms with Hippocampal Function and Schizophrenia. Neurobiol. Dis. 34, 518-524. doi:10.1016/ J.NBD.2009.03.011

Parada, G. E., Munita, R., Georgakopoulos-Soares, I., Fernandes, H. J. R., Kedlian, V. R., Metzakopian, E., et al. (2021). MicroExonator Enables Systematic Discovery and Quantification of Microexons across Mouse Embryonic Development. Genome Biol. 22, 43. doi:10.1186/s13059-020-02246-2

Parenti, I., Rabaneda, L. G., Schoen, H., and Novarino, G. (2020). Neurodevelopmental Disorders: From Genetics to Functional Pathways. Trends Neurosciences 43, 608-621. doi:10.1016/j.tins.2020.05.004

Park, H., Choi, Y., Jung, H., Kim, S., Lee, S., Han, H., et al. (2020). Splicedependent Trans-synaptic PTP $\delta$ - IL 1 RAPL 1 Interaction Regulates Synapse Formation and Non- REM Sleep. EMBO J. 39, 1-21. doi:10.15252/ embj.2019104150

Pavlowsky, A., Gianfelice, A., Pallotto, M., Zanchi, A., Vara, H., Khelfaoui, M., et al. (2010). A Postsynaptic Signaling Pathway that May Account for the Cognitive Defect Due to IL1RAPL1 Mutation. Curr. Biol. 20, 103-115. doi:10.1016/ J.CUB.2009.12.030

Pinto, D., Pagnamenta, A. T., Klei, L., Anney, R., Merico, D., Regan, R., et al. (2010). Functional Impact of Global Rare Copy Number Variation in Autism Spectrum Disorders. Nature 466, 368-372. doi:10.1038/nature09146

Piton, A., Gauthier, J., Hamdan, F. F., Lafrenière, R. G., Yang, Y., Henrion, E., et al. (2011). Systematic Resequencing of X-Chromosome Synaptic Genes in Autism Spectrum Disorder and Schizophrenia. Mol. Psychiatry 16, 867-880. doi:10.1038/mp.2010.54

Pulido, R., Serra-Pages, C., Tang, M., and Streuli, M. (1995). The LAR/PTP delta/ PTP Sigma Subfamily of Transmembrane Protein-Tyrosine-Phosphatases: Multiple Human LAR, PTP delta, and PTP Sigma Isoforms Are Expressed in a Tissue-specific Manner and Associate with the LAR-Interacting Protein LIP.1. Proc. Natl. Acad. Sci. 92, 11686-11690. doi:10.1073/pnas.92.25.11686

Quesnel-Vallières, M., Irimia, M., Cordes, S. P., and Blencowe, B. J. (2015). Essential Roles for the Splicing Regulator nSR100/SRRM4 during Nervous System Development. Genes Dev. 29, 746-759. doi:10.1101/ gad.256115.114

Roppongi, R. T., Dhume, S. H., Padmanabhan, N., Silwal, P., Zahra, N., Karimi, B., et al. (2020). LRRTMs Organize Synapses through Differential Engagement of Neurexin and PTP $\sigma$. Neuron 106, 108-125. doi:10.1016/ j.neuron.2020.01.003

Sajnani, G., Aricescu, A. R., Jones, E. Y., Gallagher, J., Alete, D., and Stoker, A. (2005). PTPo Promotes Retinal Neurite Outgrowth Non-cell-autonomously. J. Neurobiol. 65, 59-71. doi:10.1002/neu.20175

Sapieha, P. S., Duplan, L., Uetani, N., Joly, S., Tremblay, M. L., Kennedy, T. E., et al. (2005). Receptor Protein Tyrosine Phosphatase Sigma Inhibits Axon Regrowth in the Adult Injured CNS. Mol. Cell Neurosci. 28, 625-635. doi:10.1016/ j.mcn.2004.10.011
Sarhan, A. R., Patel, T. R., Cowell, A. R., Tomlinson, M. G., Hellberg, C., Heath, J. K., et al. (2016a). LAR Protein Tyrosine Phosphatase Regulates Focal Adhesions via CDK1. J. Cell Sci. 129, 2962-2971. doi:10.1242/jcs.191379

Sarhan, A. R., Patel, T. R., Creese, A. J., Tomlinson, M. G., Hellberg, C., Heath, J. K., et al. (2016b). Regulation of Platelet Derived Growth Factor Signaling by Leukocyte Common Antigen-Related (LAR) Protein Tyrosine Phosphatase: A Quantitative Phosphoproteomics Study. Mol. Cell Proteomics 15, 1823-1836. doi:10.1074/mcp.M115.053652

Schaapveld, R. Q. J., Schepens, J. T. G., Bächner, D., Attema, J., Wieringa, B., Jap, P. H. K., et al. (1998). Developmental Expression of the Cell Adhesion Moleculelike Protein Tyrosine Phosphatases LAR, RPTP $\delta$ and RPTP $\sigma$ in the Mouse. Mech. Development 77, 59-62. doi:10.1016/S0925-4773(98)00119-1

Schaapveld, R. Q. J., Schepens, J. T. G., Robinson, G. W., Attema, J., Oerlemans, F. T. J. J., Fransen, J. A. M., et al. (1997). Impaired Mammary Gland Development and Function in Mice Lacking LAR Receptor-like Tyrosine Phosphatase Activity. Developmental Biol. 188, 134-146. doi:10.1006/dbio.1997.8630

Schoch, S., Castillo, P. E., Jo, T., Mukherjee, K., Geppert, M., Wang, Y., et al. (2002). RIM1 $\alpha$ Forms a Protein Scaffold for Regulating Neurotransmitter Release at the Active Zone. Nature 415, 321-326. doi:10.1038/415321a

Schormair, B., Kemlink, D., Roeske, D., Eckstein, G., Xiong, L., Lichtner, P., et al. (2008). PTPRD (Protein Tyrosine Phosphatase Receptor Type delta) Is Associated with Restless Legs Syndrome. Nat. Genet. 40, 946-948. doi:10.1038/ng.190

Sclip, A., and Südhof, T. C. (2020). LAR Receptor Phospho-Tyrosine Phosphatases Regulate NMDA-Receptor Responses. Elife 9. doi:10.7554/ eLife.53406

Serra-Pagès, C., Kedersha, N. L., Fazikas, L., Medley, Q., Debant, A., and Streuli, M. (1995). The LAR Transmembrane Protein Tyrosine Phosphatase and a CoiledCoil LAR-Interacting Protein Co-localize at Focal Adhesions. EMBO J. 14, 2827-2838. doi:10.1002/j.1460-2075.1995.tb07282.x

Serra-Pagès, C., Medley, Q. G., Tang, M., Hart, A., and Streuli, M. (1998). Liprins, a Family of LAR Transmembrane Protein-Tyrosine Phosphatase-Interacting Proteins. J. Biol. Chem. 273, 15611-15620. doi:10.1074/jbc.273.25.15611

Serra-Pagès, C., Saito, H., and Streuli, M. (1994). Mutational Analysis of Proprotein Processing, Subunit Association, and Shedding of the LAR Transmembrane Protein Tyrosine Phosphatase. J. Biol. Chem. 269, 23632-23641.

Shen, Y., Tenney, A. P., Busch, S. A., Horn, K. P., Cuascut, F. X., Liu, K., et al. (2009). PTP $\sigma$ Is a Receptor for Chondroitin Sulfate Proteoglycan, an Inhibitor of Neural Regeneration. Science 326 (5952), 592-596. doi:10.1126/ science. 1178310

Shijo, T., Warita, H., Suzuki, N., Kitajima, Y., Ikeda, K., Akiyama, T., et al. (2018). Aberrant Astrocytic Expression of Chondroitin Sulfate Proteoglycan Receptors in a Rat Model of Amyotrophic Lateral Sclerosis. J. Neuro Res. 96, 222-233. doi:10.1002/jnr.24127

Simó, S., and Cooper, J. A. (2012). Regulation of Dendritic Branching by Cdc42 GAPs: Figure 1. Genes Dev. 26, 1653-1658. doi:10.1101/gad.199034.112

Siu, R., Fladd, C., and Rotin, D. (2007). N-cadherin Is an In Vivo Substrate for Protein Tyrosine Phosphatase Sigma (РTPб) and Participates in РTP $\sigma$ Mediated Inhibition of Axon Growth. Mol. Cell. Biol. 27, 208-219. doi:10.1128/mcb.00707-06

Song, Y. S., Lee, H.-J., Prosselkov, P., Itohara, S., and Kim, E. (2013). Trans-induced Cis Interaction in the Tripartite NGL-1, Netrin-G1, and LAR Adhesion Complex Promotes Excitatory Synaptic Development. J. Cell Sci. 126, 4926-4938. doi:10.1242/jcs.129718

Spangler, S. A., and Hoogenraad, C. C. (2007). Liprin- $\alpha$ Proteins: Scaffold Molecules for Synapse Maturation. Biochem. Soc. Trans. 35, 1278-1282. doi:10.1042/BST0351278

Stewart, K., Uetani, N., Hendriks, W., Tremblay, M. L., Bouchard, M., Kuwaki, T., et al. (2013). Inactivation of LAR Family Phosphatase Genes Ptprs and Ptprf Causes Craniofacial Malformations Resembling Pierre-Robin Sequence. Development 140, 3413-3422. doi:10.1242/dev.094532

Stoker, A. W. (2015). RPTPs in Axons, Synapses and Neurology. Semin. Cell Developmental Biol. 37, 90-97. doi:10.1016/j.semcdb.2014.09.006

Streuli, M., Krueger, N. X., Ariniello, P. D., Tang, M., Munro, J. M., Blattler, W. A., et al. (1992). Expression of the Receptor-Linked Protein Tyrosine Phosphatase LAR: Proteolytic Cleavage and Shedding of the CAM-like Extracellular Region. EMBO J. 11, 897-907. doi:10.1002/j.14602075.1992.tb05128.x 
Streuli, M., Krueger, N. X., Thai, T., Tang, M., and Saito, H. (1990). Distinct Functional Roles of the Two Intracellular Phosphatase like Domains of the Receptor-Linked Protein Tyrosine Phosphatases LCA and LAR. EMBO J. 9, 2399-2407. doi:10.1002/j.1460-2075.1990.tb07415.x

Sun, Q. L., Wang, J., Bookman, R. J., and Bixby, J. L. (2000). Growth Cone Steering by Receptor Tyrosine Phosphatase $\delta$ Defines a Distinct Class of Guidance Cue. Mol. Cell Neurosci. 16, 686-695. doi:10.1006/mcne.2000.0893

Takahashi, H., Arstikaitis, P., Prasad, T., Bartlett, T. E., Wang, Y. T., Murphy, T. H., et al. (2011). Postsynaptic TrkC and Presynaptic PTPo Function as a Bidirectional Excitatory Synaptic Organizing Complex. Neuron 69, 287-303. doi:10.1016/j.neuron.2010.12.024

Takahashi, H., and Craig, A. M. (2013). Protein Tyrosine Phosphatases PTP $\delta$, PTP $\sigma$, and LAR: Presynaptic Hubs for Synapse Organization. Trends Neurosciences 36, 522-534. doi:10.1016/j.tins.2013.06.002

Takahashi, H., Katayama, K.-i., Sohya, K., Miyamoto, H., Prasad, T., Matsumoto, Y., et al. (2012). Selective Control of Inhibitory Synapse Development by Slitrk3-Ptp $\delta$ Trans-synaptic Interaction. Nat. Neurosci. 15, 389-398. doi: $10.1038 / \mathrm{nn} .3040$

The Hd iPSC Consortium (2012). Induced Pluripotent Stem Cells from Patients with Huntington's Disease Show CAG-Repeat-ExpansionAssociated Phenotypes. Cell Stem Cell 11, 264-278. doi:10.1016/ j.stem.2012.04.027

Thompson, K., Uetani, N., Manitt, C., Elchebly, M., Tremblay, M. L., and Kennedy, T. E. (2003). Receptor Protein Tyrosine Phosphatase Sigma Inhibits Axonal Regeneration and the Rate of Axon Extension. Mol. Cell Neurosci. 23, 681-692. doi:10.1016/S1044-7431(03)00120-9

Tisi, M. A., Xie, Y., Yeo, T. T., and Longo, F. M. (2000). Downregulation of LAR Tyrosine Phosphatase Prevents Apoptosis and Augments NGF-Induced Neurite Outgrowth. J. Neurobiol., 42, 477-486. doi:10.1002/(sici)10974695(200003)42:4<477::aid-neu8>3.0.co;2-b

Tomita, H., Cornejo, F., Aranda-Pino, B., Woodard, C. L., Rioseco, C. C., Neel, B. G., et al. (2020). The Protein Tyrosine Phosphatase Receptor Delta Regulates Developmental Neurogenesis. Cell Rep. 30, 215-228. doi:10.1016/ j.celrep.2019.11.033

Tonks, N. K., Diltz, C. D., and Fischer, E. H. (1988). Purification of the Major Protein-Tyrosine-Phosphatases of Human Placenta. J. Biol. Chem. 263, 6722-6730. doi:10.1016/s0021-9258(18)68702-2

Tonks, N. K. (2006). Protein Tyrosine Phosphatases: From Genes, to Function, to Disease. Nat. Rev. Mol. Cell Biol. 7, 833-846. doi:10.1038/nrm2039

Uetani, N., Bertozzi, K., Chagnon, M. J., Hendriks, W., Tremblay, M. L., and Bouchard, M. (2009). Maturation of Ureter-Bladder Connection in Mice Is Controlled by LAR Family Receptor Protein Tyrosine Phosphatases. J. Clin. Invest. 119, 924-935. doi:10.1172/JCI37196

Uetani, N., Chagnon, M. J., Kennedy, T. E., Iwakura, Y., and Tremblay, M. L. (2006). Mammalian Motoneuron Axon Targeting Requires Receptor Protein Tyrosine Phosphatases and. J. Neurosci. 26, 5872-5880. doi:10.1523/ JNEUROSCI.0386-06.2006

Uetani, N., Kato, K., Ogura, H., Mizuno, K., Kawano, K., Mikoshiba, K., et al. (2000). Impaired Learning with Enhanced Hippocampal Long-Term Potentiation in PTPdelta-Deficient Mice. EMBO J. 19, 2775-2785. doi:10.1093/emboj/19.12.2775

Uhl, G. R., Drgon, T., Johnson, C., Fatusin, O. O., Liu, Q.-R., Contoreggi, C., et al. (2008a). "Higher Order" Addiction Molecular Genetics: Convergent Data from Genome-wide Association in Humans and Mice. Biochem. Pharmacol. 75, 98-111. doi:10.1016/J.BCP.2007.06.042

Uhl, G. R., Drgon, T., Johnson, C., Ramoni, M. F., Behm, F. M., and Rose, J. E. (2010). Genome-wide Association for Smoking Cessation success in a Trial of Precessation Nicotine Replacement. Mol. Med. 16, 513-526. doi:10.2119/ molmed.2010.00052

Uhl, G. R., Liu, Q.-R., Drgon, T., Johnson, C., Walther, D., Rose, J. E., et al. (2008b). Molecular Genetics of Successful Smoking Cessation. Arch. Gen. Psychiatry 65, 683-693. doi:10.1001/archpsyc.65.6.683

Um, J. W., Kim, K. H., Park, B. S., Choi, Y., Kim, D., Kim, C. Y., et al. (2014). Structural Basis for LAR-RPTP/Slitrk Complex-Mediated Synaptic Adhesion. Nat. Commun. 5, 5423. doi:10.1038/ncomms6423

Um, J. W., and Ko, J. (2013). LAR-RPTPs: Synaptic Adhesion Molecules that Shape Synapse Development. Trends Cell Biol. 23, 465-475. doi:10.1016/ j.tcb.2013.07.004
Valnegri, P., Montrasio, C., Brambilla, D., Ko, J., Passafaro, M., and Sala, C. (2011). The X-Linked Intellectual Disability Protein IL1RAPL1 Regulates Excitatory Synapse Formation by Binding PTP $\delta$ and RhoGAP2. Hum. Mol. Genet. 20, 4797-4809. doi:10.1093/hmg/ddr418

Van der Zee, C. E. E. M., Man, T. Y., Van Lieshout, E. M. M., Van der Heijden, I., Van Bree, M., and Hendriks, W. J. A. J. (2003). Delayed Peripheral Nerve Regeneration and central Nervous System Collateral Sprouting in Leucocyte Common Antigen-Related Protein Tyrosine Phosphatase-Deficient Mice. Eur. J. Neurosci. 17, 991-1005. doi:10.1046/j.1460-9568.2003.02516.x

Van Lieshout, E. M. M., Van der Heijden, I., Hendriks, W. J. A. J., and Van der Zee, C. E. E. M. (2001). A Decrease in Size and Number of Basal Forebrain Cholinergic Neurons Is Paralleled by Diminished Hippocampal Cholinergic Innervation in Mice Lacking Leukocyte Common Antigen-Related Protein Tyrosine Phosphatase Activity. Neuroscience 102, 833-841. doi:10.1016/S03064522(00)00526-1

von Schantz, C., Saharinen, J., Kopra, O., Cooper, J. D., Gentile, M., Hovatta, I., et al. (2008). Brain Gene Expression Profiles of Cln1 and Cln5 Deficient Mice Unravels Common Molecular Pathways Underlying Neuronal Degeneration in NCL Diseases. BMC Genomics 9, 146. doi:10.1186/1471-2164-9-146

Wallace, M. J., Batt, J., Fladd, C. A., Henderson, J. T., Skarnes, W., and Rotin, D. (1999). Neuronal Defects and Posterior Pituitary Hypoplasia in Mice Lacking the Receptor Tyrosine Phosphatase РТР $\sigma$. Nat. Genet. 21, 334-338. doi:10.1038/6866

Wallace, M. J., Fladd, C., Batt, J., and Rotin, D. (1998). The Second Catalytic Domain of Protein Tyrosine Phosphatase $\delta$ (РTP $\delta$ ) Binds to and Inhibits the First Catalytic Domain of PTP५. Mol. Cell. Biol. 18, 2608-2616. doi:10.1128/ MCB.18.5.2608

Wamsley, B., Jaglin, X. H., Favuzzi, E., Quattrocolo, G., Nigro, M. J., Yusuf, N., et al. (2018). Rbfox1 Mediates Cell-type-specific Splicing in Cortical Interneurons. Neuron 100 (4), 846-859. doi:10.1016/j.neuron.2018.09.026

Wehrle-Haller, B. (2012). Assembly and Disassembly of Cell Matrix Adhesions. Curr. Opin. Cell Biol. 24 (5), 569-581. doi:10.1016/j.ceb.2012.06.010

Weng, Y.-L., Liu, N., DiAntonio, A., and Broihier, H. T. (2011). The Cytoplasmic Adaptor Protein Caskin Mediates Lar Signal Transduction during Drosophila Motor Axon Guidance. J. Neurosci. 31, 4421-4433. doi:10.1523/ JNEUROSCI.5230-10.2011

Weyn-Vanhentenryck, S. M., Feng, H., Ustianenko, D., Duffié, R., Yan, Q., Jacko, M., et al. (2018). Precise Temporal Regulation of Alternative Splicing during Neural Development. Nat. Commun. 9, 1-17. doi:10.1038/s41467-018-04559-0

Wills, Z., Bateman, J., Korey, C. A., Comer, A., and Van Vactor, D. (1999). The Tyrosine Kinase Abl and its Substrate Enabled Collaborate with the Receptor Phosphatase Dlar to Control Motor Axon Guidance. Neuron 22, 301-312. doi:10.1016/S0896-6273(00)81091-0

Winkelmann, J., Czamara, D., Schormair, B., Knauf, F., Schulte, E. C., Trenkwalder, C., et al. (2011). Genome-Wide Association Study Identifies Novel Restless Legs Syndrome Susceptibility Loci on 2p14 and 16q12.1. Plos Genet. 7, e1002171. doi:10.1371/journal.pgen.1002171

Won, S. Y., and Kim, H. M. (2018). Structural Basis for LAR-RPTP-Mediated Synaptogenesis. Mol. Cell 41, 622-630. doi:10.14348/molcells.2018.0202

Won, S. Y., Kim, C. Y., Kim, D., Ko, J., Um, J. W., Lee, S. B., et al. (2017). LARRPTP Clustering Is Modulated by Competitive Binding between Synaptic Adhesion Partners and Heparan Sulfate. Front. Mol. Neurosci. 10, 327. doi:10.3389/fnmol.2017.00327

Woo, J., Kwon, S.-K., Choi, S., Kim, S., Lee, J.-R., Dunah, A. W., et al. (2009). Transsynaptic Adhesion between NGL-3 and LAR Regulates the Formation of Excitatory Synapses. Nat. Neurosci. 12, 428-437. doi:10.1038/nn.2279

Wu, K., Hanna, G. L., Easter, P., Kennedy, J. L., Rosenberg, D. R., and Arnold, P. D. (2013). Glutamate System Genes and Brain Volume Alterations in Pediatric Obsessive-Compulsive Disorder: A Preliminary Study. Psychiatry Res. Neuroimaging 211, 214-220. doi:10.1016/J.PSCYCHRESNS.2012.07.003

Wyszynski, M., Kim, E., Dunah, A. W., Passafaro, M., Valtschanoff, J. G., SerraPagès, C., et al. (2002). Interaction between GRIP and Liprin- $\alpha /$ SYD2 Is Required for AMPA Receptor Targeting. Neuron 34, 39-52. doi:10.1016/ S0896-6273(02)00640-2

Xie, X., Luo, L., Liang, M., Zhang, W., Zhang, T., Yu, C., et al. (2020). Structural Basis of Liprin- $\alpha$-Promoted LAR-RPTP Clustering for Modulation of Phosphatase Activity. Nat. Commun. 11, 1-12. doi:10.1038/s41467-01913949-x 
Xie, Y., Massa, S. M., Ensslen-Craig, S. E., Major, D. L., Yang, T., Tisi, M. A., et al. (2006). Protein-tyrosine Phosphatase (PTP) Wedge Domain Peptides. J. Biol. Chem. 281, 16482-16492. doi:10.1074/jbc.M603131200

Yamagata, A., Yoshida, T., Sato, Y., Goto-Ito, S., Uemura, T., Maeda, A., et al. (2015). Mechanisms of Splicing-dependent Trans-synaptic Adhesion by PTP $\delta$ IL1RAPL1/IL-1RAcP for Synaptic Differentiation. Nat. Commun. 6, 1-11. doi:10.1038/ncomms7926

Yang, Q., Li, L., Yang, R., Shen, G.-Q., Chen, Q., Foldvary-Schaefer, N., et al. (2011). Family-based and Population-Based Association Studies Validate PTPRD as a Risk Factor for Restless Legs Syndrome. Mov. Disord. 26, 516-519. doi:10.1002/ mds.23459

Yang, T., Bernabeu, R., Xie, Y., Zhang, J. S., Massa, S. M., Rempel, H. C., et al. (2003). Leukocyte Antigen-Related Protein Tyrosine Phosphatase Receptor: a Small Ectodomain Isoform Functions as a Homophilic Ligand and Promotes Neurite Outgrowth. J. Neurosci. 23, 3353-3363. doi:10.1523/JNEUROSCI.2308-03353.2003

Yang, T., Yin, W., Derevyanny, V. D., Moore, L. A., and Longo, F. M. (2005). Identification of an Ectodomain within the LAR Protein Tyrosine Phosphatase Receptor that Binds Homophilically and Activates Signalling Pathways Promoting Neurite Outgrowth. Eur. J. Neurosci. 22, 2159-2170. doi:10.1111/ j.1460-9568.2005.04403.x

Yeo, T. T., Yang, T., Massa, S. M., Zhang, J. S., Honkaniemi, J., Butcher, L. L., et al. (1997). Deficient LAR Expression Decreases Basal Forebrain Cholinergic Neuronal Size and Hippocampal Cholinergic Innervation. J. Neurosci. Res. 47, 348-360. doi:10.1002/(sici)1097-4547(19970201)47:3<348::aid-jnr13>3.0.co;2-y

Yim, Y. S., Kwon, Y., Nam, J., Yoon, H. I., Lee, K., Kim, D. G., et al. (2013). Slitrks Control Excitatory and Inhibitory Synapse Formation with LAR Receptor Protein Tyrosine Phosphatases. Proc. Natl. Acad. Sci. 110, 4057-4062. doi:10.1073/pnas.1209881110

Yoshida, T., Shiroshima, T., Lee, S.-J., Yasumura, M., Uemura, T., Chen, X., et al. (2012). Interleukin-1 Receptor Accessory Protein Organizes Neuronal Synaptogenesis as a Cell Adhesion Molecule. J. Neurosci. 32, 2588-2600. doi:10.1523/JNEUROSCI.4637-11.2012

Yoshida, T., Yamagata, A., Imai, A., Kim, J., Izumi, H., Nakashima, S., et al. (2021). Canonical versus Non-canonical Transsynaptic Signaling of Neuroligin 3
Tunes Development of Sociality in Mice. Nat. Commun. 12 (1), 1848. doi:10.1038/s41467-021-22059-6

Yoshida, T., Yasumura, M., Uemura, T., Lee, S.-J., Ra, M., Taguchi, R., et al. (2011). IL-1 Receptor Accessory Protein-like 1 Associated with Mental Retardation and Autism Mediates Synapse Formation by Trans-synaptic Interaction with Protein Tyrosine Phosphatase. J. Neurosci. 31, 13485-13499. doi:10.1523/ JNEUROSCI.2136-11.2011

Young, K. A., Biggins, L., and Sharpe, H. J. (2021). Protein Tyrosine Phosphatases in Cell Adhesion. Biochem. J. 478, 1061-1083. doi:10.1042/BCJ20200511

Youngs, E. L., Henkhaus, R., Hellings, J. A., and Butler, M. G. (2012). IL1RAPL1 Gene Deletion as a Cause of X-Linked Intellectual Disability and Dysmorphic Features. Eur. J. Med. Genet. 55, 32-36. doi:10.1016/ J.EJMG.2011.08.004

Zhu, Q., Tan, Z., Zhao, S., Huang, H., Zhao, X., Hu, X., et al. (2015). Developmental Expression and Function Analysis of Protein Tyrosine Phosphatase Receptor Type D in Oligodendrocyte Myelination. Neuroscience 308, 106-114. doi:10.1016/J.NEUROSCIENCE.2015.08.062

Conflict of Interest: The authors declare that the research was conducted in the absence of any commercial or financial relationships that could be construed as a potential conflict of interest.

Publisher's Note: All claims expressed in this article are solely those of the authors and do not necessarily represent those of their affiliated organizations, or those of the publisher, the editors and the reviewers. Any product that may be evaluated in this article, or claim that may be made by its manufacturer, is not guaranteed or endorsed by the publisher.

Copyright (C) 2021 Cornejo, Cortés, Findlay and Cancino. This is an open-access article distributed under the terms of the Creative Commons Attribution License (CC $B Y)$. The use, distribution or reproduction in other forums is permitted, provided the original author(s) and the copyright owner(s) are credited and that the original publication in this journal is cited, in accordance with accepted academic practice. No use, distribution or reproduction is permitted which does not comply with these terms. 